\title{
Recovery of Underwater Visibility and Structure by Polarization Analysis
}

\author{
Yoav Y. Schechner and Nir Karpel
}

\begin{abstract}
Underwater imaging is important for scientific research and technology as well as for popular activities, yet it is plagued by poor visibility conditions. In this paper, we present a computer vision approach that removes degradation effects in underwater vision. We analyze the physical effects of visibility degradation. It is shown that the main degradation effects can be associated with partial polarization of light. Then, an algorithm is presented, which inverts the image formation process for recovering good visibility in images of scenes. The algorithm is based on a couple of images taken through a polarizer at different orientations. As a by-product, a distance map of the scene is also derived. In addition, this paper analyzes the noise sensitivity of the recovery. We successfully demonstrated our approach in experiments conducted in the sea. Great improvements of scene contrast and color correction were obtained, nearly doubling the underwater visibility range.
\end{abstract}

Index Terms-Color, illumination, image enhancement, inverse problems, polarized light, scattering, three-dimensional reconstruction, undersea vision, underwater imaging.

\section{UNDERWATER VISION}

$\mathbf{U}$ NDERWATER vision is plagued by poor visibility conditions [1]-[6]. According to [7], most computer vision methods (e.g., those based on stereo triangulation or on structure from motion) cannot be employed directly underwater. This is due to the particularly challenging environmental conditions that complicate image matching and analysis. It is important to alleviate these visibility problems since underwater imaging is widely used in scientific research and technology. Computer vision methods are being used in this mode of imaging for various applications [5], [6], [8]-[16] such as mine detection, inspection of underwater power and telecommunication cables, pipelines [7], [17], nuclear reactors, and columns of offshore platforms [7]. Underwater computer vision is used commercially to help swimming pool lifeguards [18]. As in conventional computer vision, algorithms are sought for navigation and control [19] of submerged robots. In addition, underwater imaging is used for research in marine biology [6], [20]-[22], archaeology [1], [23]-[25], and mapping [19]. Moreover, underwater photography [26], [27] is becoming more accessible to a wider public.

Manuscript received May 5, 2004; revised December 14, 2004; accepted March 5, 2005. Y. Schechner is a Landau Fellow - supported by the Taub Foundation. This paper was supported by the Israeli Science Foundation (grant No. 315/04) and by the Ollendorff Minerva Center in the Electrical Engineering Department at the Technion. Minerva is funded through the BMBF. Associate Editor: A. Trucco.

The authors are with the Department of Electrical Engineering, Technion-Israel Institute of Technology, Haifa 32000, Israel (e-mail: yoav@ee.technion.ac.il; karpeln@ netvision.net.il).

Digital Object Identifier 10.1109/JOE.2005.850871

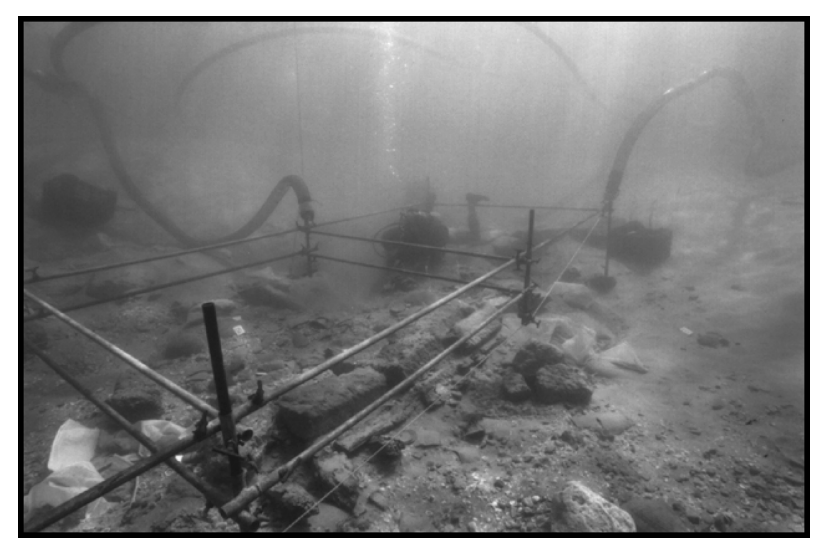

Fig. 1. Underwater Mediterranean archaeological site. Visibility degrades quickly as a function of distance.

What makes underwater imaging so problematic? To understand the challenge, consider Fig. 1. which shows an underwater archaeological site about $2.5-\mathrm{m}$ deep. It is easy to see that visibility degradation effects vary as distances to objects increase [3], [28]. Since objects in the field of view (FOV) are at different distances from the camera, the causes for image degradation are spatially varying. This situation is analogous to open-air vision in bad weather (fog or haze) described in [29]-[34]. Contrary to this fact, traditional image enhancement tools, e.g., high pass filtering and histogram equalization, are typically spatially invariant. Since they do not model the spatially varying distance dependencies, traditional methods are of limited utility in countering visibility problems, as has been demonstrated in past experiments [33], [35] as well as in this paper.

In this paper, we develop a physics-based approach for recovery of visibility when imaging underwater scenes in natural illumination. Since it is based on the models of image formation, the approach automatically accounts for dependencies on object distance and estimates a distance map of the scene as a by-product. The approach is fast and relies on raw images taken through different states of a polarizing filter. ${ }^{1}$ These raw images have slight photometric differences with respect to one another. The differences serve as initial cues for our algorithm factoring out turbidity effects. It is interesting to note that marine animals use polarization for improved vision [6], [21], [22], [40]-[48].

To demonstrate the approach, we built an underwater polarization imaging system composed of both custom and off-theshelf components (the considerations for selecting the components are described). We used the method by experimenting in

${ }^{1}$ Polarization-filtered images have been used in various computer vision algorithms dealing with reflections [36]-[38]. These methods evolved along with developments of polarimetric imaging devices [6], [39]. 


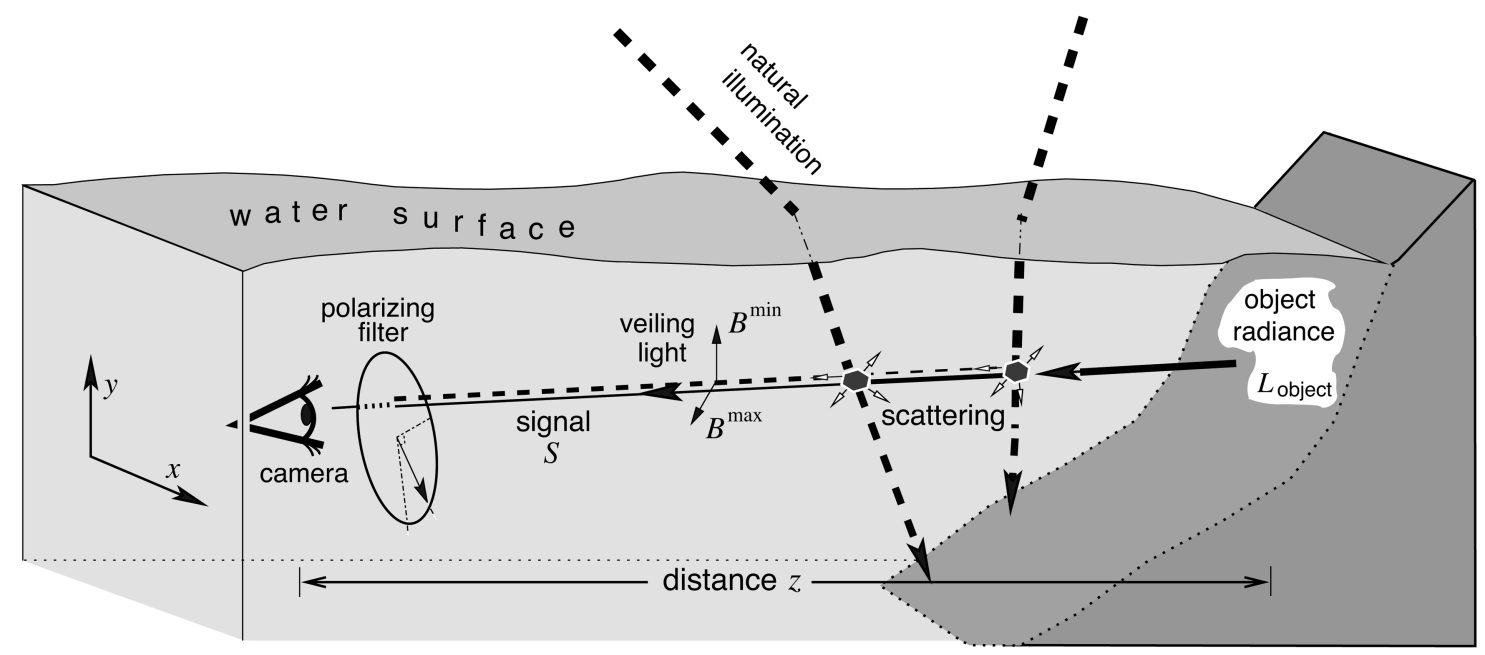

Fig. 2. Underwater imaging of a scene through a polarizing filter. (Dashed rays) Illumination enters the water through Snell's window. Light is scattered towards the camera by particles in the water, creating veiling light. Veiling light increases with distance $z$ to the object. (Solid ray) Light emanating from the object is attenuated and somewhat blurred as $z$ increases, leading to the signal $S$. The partial polarization of veiling light is significant, contrary to the signal. Without scattering and absorption along the LOS, the object radiance would have been $L_{\text {object }}$.

the sea. Significant improvements of contrast and color are obtained. The recovered range maps indicate that the visibility range has been approximately doubled by the approach. Partial preliminary results were presented in [34].

\section{Relation to Previous Methods}

A common approach to improve underwater visibility and color is based on artificial illumination. The most popular realization of this approach uses an off axis widefield strobe [26], [27] attached to the camera. In practice, this method is limited to photography of objects that are relatively close to the camera [26], [27]. This is the result of the falloff of scene irradiance with the distance from the strobe. Moreover, "sea snow" may be created by the defocus blur of the strong backscatter from suspended particles at close distances [26], [27].

To bypass the backscatter problem, advanced research underwater imaging systems use specialized active radiation hardware [2]-[4], [12], [16], [49]-[53]. Yet, the range of such systems is limited for the reason mentioned with respect to a widefield torch: at some distance, the source's falloff leads to too low levels of scene irradiance. To handle this problem, such systems tend to be highly power consuming, complex, and expensive. To avoid these aspects, in this article we deal with a complementary approach: passive computer vision that exploits natural illumination. When available, natural illumination exists all over the scene, alleviating the need to project energy towards objects.

It was demonstrated decades ago [54] that polarization filtering can enhance contrast in passive underwater vision. Yet, simple optical filtering [54], [55] may have a limited ${ }^{2}$ effect, indicating that some postprocessing is needed, based on the acquisition of both components of polarized light [54]. One approach is based on a simple subtraction of the differently polarization-filtered images [2], [39], or displays the degree of polarization (DOP) [5], [46]. That approach has fundamental dis-

\footnotetext{
${ }^{2}$ The experiments described in [55] were based on subjective responses of divers, rather than on photographs. They used polarizing filters having an extinction ratio of 1:2. This ratio is much worse than the current state of the art.
}

advantages. It assumes that polarization is associated with the object radiation rather than the causes that degrade the signal. However, due to depolarization [14], that assumption becomes invalid as distances increase. Our approach is based on a contrary fact [43], [54], [56], [57]: in natural illumination, underwater polarization is associated with the prime visibility disturbance that we wish to delete (veiling light). This observation has recently been utilized to enhance visibility in a perpendicular illumination setting [58]. Nevertheless, enhancement methods such as those used in [2], [5], [39], [46], and [58] are far from inverting the image formation process and recovering the objects. In contrast, our approach inverts the physical model in general natural lighting, thus the recovered image is similar to clear visibility appearance.

\section{IMAGE COMPONENTS}

This section describes the model of image formation. It describes the assumptions the paper is based upon. This section also details the approximations we make and the insights behind them. In addition, this section presents the notations we use. As depicted in Fig. 2, when imaging underwater we sense two sources. The first source is the scene object at distance $z$, whose radiance is attenuated by absorption and scattering in the water. It is also somewhat blurred. The image corresponding to this degraded source is the signal. The second source is the ambient illumination. Part of the ambient light is scattered towards the camera by the particles in the water and is termed in the literature as veiling light [43], [48], [59]-[63], path radiance [64], [65], spacelight [41], [43], [48], [54], [59], and backscatter [3], [66]. The veiling light is partially polarized. This fact is used by our visibility restoration algorithm. We now describe each of these components.

\section{A. The Signal}

1) Direct Transmission: The signal is composed of two components termed direct transmission and forward scattering [3], [66], [67]. This section details the direct transmission 
while the next section describes forward scattering. As a light ray progresses from the object towards the camera, part of its energy is lost due to scattering and absorption. The fraction that does reach the camera is the direct transmission ${ }^{3}$ given by

$$
D(x, y)=L_{\text {object }}(x, y) e^{-c z},
$$

where $z$ is the distance to the object, which depends on the pixel coordinates $x$ and $y$, while $c$ is the attenuation coefficient. Here, $L_{\text {object }}$ is the object radiance we would have sensed had there been no scattering and absorption along the line of sight (LOS).

The attenuation coefficient is given by $c=a+b$, where $a$ is the absorption coefficient and $b$ is the total scattering coefficient of the water. The scattering coefficient $b$ expresses the ability of an infinitesimal water volume to scatter flux in all directions. Integrating over all solid angles $\boldsymbol{\Theta}$

$$
b=\int_{\boldsymbol{\Theta}} b(\boldsymbol{\Theta}) d \Omega=2 \pi \int_{0}^{\pi} b(\theta) \sin (\theta) d \theta
$$

where $\theta$ is the scattering angle relative to the propagation direction. The angular scattering coefficient $b(\theta)$ is sometimes referred to as the phase function [57]. Note that the variables $a, b(\theta), c$, and $L_{\text {object }}$ are all functions of the wavelength $\lambda$.

2) Forward Scattering: The forward scattering component is similar to the direct transmission. However, it represents light scattered forward at small angles relative to the LOS. This creates image blur given by the convolution

$$
F(x, y)=D(x, y) * g_{z}(x, y)
$$

where $D$ is given by (1) and $g_{z}$ is a point spread function (PSF). The PSF is parameterized by the distance $z$, since the farther the object the wider the support of the blur kernel.

There are several models in the literature for the form of the underwater PSF [66], [68]. Since the PSF depends on the hydrosols suspended in the water, the models are typically parameterized by various empirical constants. For example, the model in [3] and [66] is of the form

$$
g_{z}=\left(e^{-\gamma z}-e^{-c z}\right) \mathcal{F}^{-1}\left\{G_{z}\right\} \quad \text { where } \quad G_{z}=e^{-K z \omega},
$$

where $K>0$ and $\gamma$ are empirical constants, $\mathcal{F}^{-1}$ is the inverse Fourier transform, and $\omega$ is the spatial frequency in the image plane. Note that $G_{z}$ is a low pass filter. The effective frequency "width" of $G_{z}$ is inversely proportional to $z$. This expresses the increase of spatial blur spread for distant objects. The constant $\gamma$ is limited to $|\gamma| \leq c$ [66]. It is important to note that, according to the models obtained empirically and through numerical simulations [66], [68], the PSF does not conserve energy while light propagates in $z$. This is clearly the case in (4). Thus, forward scattering is a blurred and attenuated version of $D$.
Accounting for both the direct transmission (1) and the forward scattering (3), we define the signal as

$$
S=D+F
$$

We define an effective object radiance $L_{\text {object }}^{\text {effective }}$ as

$$
L_{\text {object }}^{\text {effective }}=L_{\text {object }}+L_{\text {object }} * g_{z} \text {. }
$$

It is a somewhat blurred version of $L_{\text {object }}$. From (1), (3), and (5), the signal is

$$
S=e^{-c z} L_{\text {object }}^{\text {effective }} \text {. }
$$

In Section III-C, we claim and demonstrate that in practice blur is not the prime cause of underwater image degradation.

\section{B. Veiling Light}

Veiling light does not originate from the object on the LOS. Rather, light coming from ambient illumination sources is scattered towards the camera (Fig. 2). The LOS is naturally lit mostly from the water surface above. In addition, the LOS is illuminated by the sea bottom and by scattering particles in the surrounding water volume. Before integrating all the contributions, let us first analyze the effect of a single distant source.

The source illuminates the particles on the LOS from a direction $\boldsymbol{\Theta}=(\theta, \varphi)$ relative to the LOS with intensity $I^{\text {source }}$. Following [3] and [66], the contribution of this source to the veiling light is

$$
B(\boldsymbol{\Theta})=\int_{0}^{z} b(\theta) I^{\text {source }}(\boldsymbol{\Theta}) e^{-c l}\left[1-\frac{f}{\left(l+l_{0}\right)}\right]^{2} d l
$$

where $f$ is the focal length of the camera and $l_{0}$ is the distance between the lens and the underwater housing port. This integral accounts for scattering into the LOS at some distance $l$ followed by attenuation until reaching the camera. It also accounts for the geometric projection of the irradiance on the detector via the ratio $f /\left(l+l_{0}\right)$.

It is shown that we can considerably simplify the expression for veiling light relative to (8). We can do this because typically $f /\left(c^{-1}+l_{0}\right)<<1$. Consider typical ranges of values as $c^{-1} \in[3,10 \mathrm{~m}]$ (according to [57]), $f \in[20,50 \mathrm{~mm}]$, $l_{0} \approx 80 \mathrm{~mm}$, and object distance in the order of meters. We assessed the integral numerically. It is shown in Fig. 3 that to an accuracy of $99 \%,(8)$ can be written as

$$
B(\boldsymbol{\Theta})_{\text {approx }} \approx \kappa(f) b(\theta) I^{\text {source }}(\boldsymbol{\Theta}) \int_{0}^{z} e^{-c l} d l,
$$

where the correction coefficient is given by

$$
\kappa=\frac{B(\boldsymbol{\Theta})_{\text {approx }}}{B(\boldsymbol{\Theta})}
$$




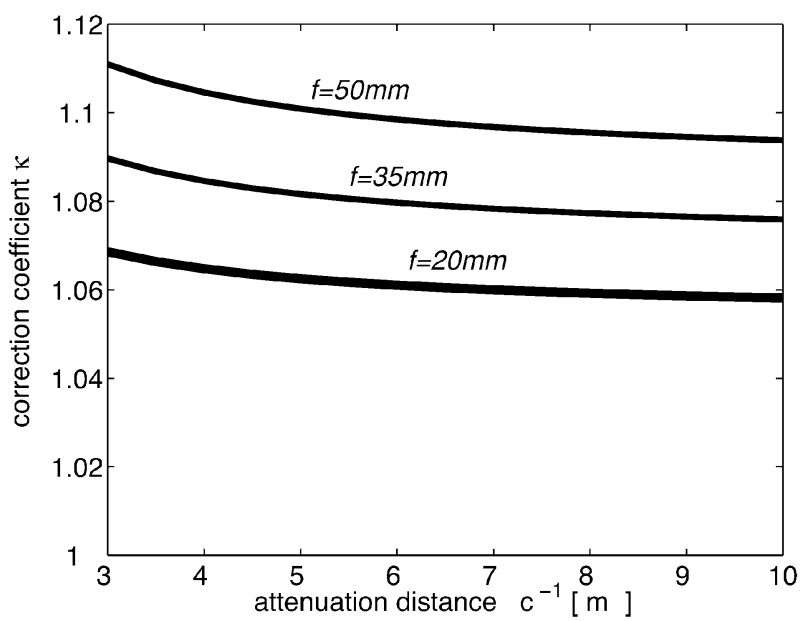

Fig. 3. Numerical assessment of the correction coefficient $\kappa$ as a function of focal length $f$ and attenuation coefficient $c$.

For instance, as shown in Fig. 3, a focal length of $f=20 \mathrm{~mm}$ yields $\kappa \approx 1.06$.

Solving the integral in (9) yields

$$
B(\boldsymbol{\Theta})_{\operatorname{approx}}=B_{\infty}(\boldsymbol{\Theta})\left(1-e^{-c z}\right) .
$$

This close-form expression is much simpler than (8). It is easily seen that the farther the object the larger the veiling light is. In (11), the variables

$$
B_{\infty}(\boldsymbol{\Theta}) \equiv \kappa I^{\text {source }}(\boldsymbol{\Theta}) b(\theta)
$$

expresses the veiling light in an LOS that extends to infinity in the water. Summing up the contribution from light sources at all directions, the total veiling light is

$$
B(x, y)=\int_{\Theta} B(\boldsymbol{\Theta})_{\text {approx }} d \boldsymbol{\Theta}=B_{\infty}\left(1-e^{-c z}\right),
$$

where

$$
B_{\infty} \equiv \int_{\Theta} B_{\infty}(\boldsymbol{\Theta}) d \boldsymbol{\Theta}
$$

is a scalar termed the water background [43], [54] that depends on $\lambda$. The veiling light $B$ implicitly depends on $x$ and $y$ due to its explicit dependence on the distance $z$.

Fig. 4 depicts the scattering of light towards the LOS by particles close to it. It shows that this light can be represented as originating from equivalent sources at infinity. This equivalence is based on an assumption of homogeneous lighting along the LOS. We believe that this is a reasonable assumption in horizontal photography. The reason is that underwater lighting naturally comes from a limited light cone directly above [21], [47], [65] and is thus typically unobscured along the LOS. Thanks to this equivalence, expression (13), which was developed for distant light sources, is applicable to the general case of scattering from nondistant particles suspended in the water volume.

\section{The Dominant Degradation Component}

We now discuss the contribution of each of the above-mentioned components. In particular, we claim that image blur is not the dominant cause for image contrast degradation. Rather,
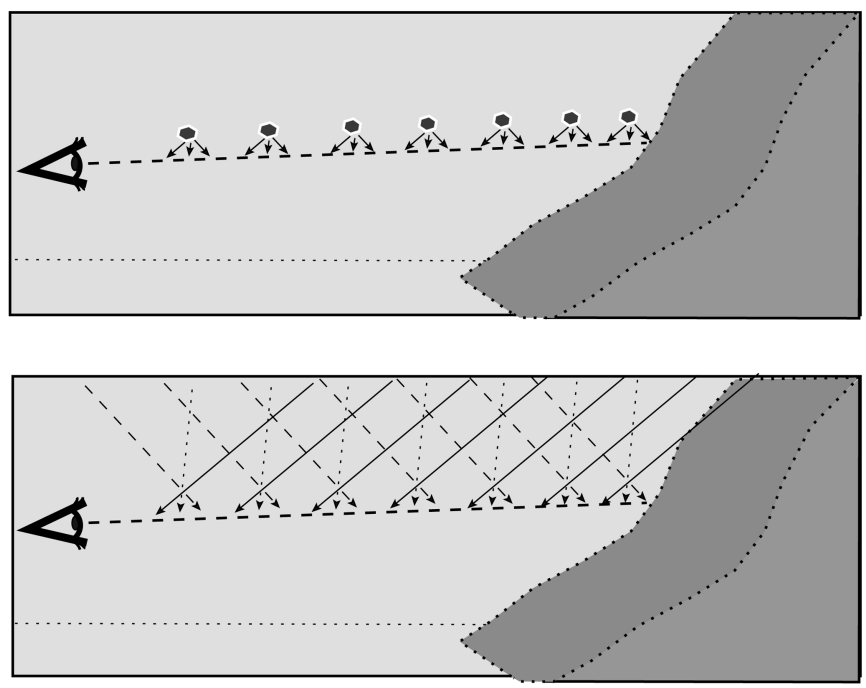

Fig. 4. (Top) Light scattered towards the LOS by nearby particles. (Bottom) Light emanating from nearby particles can be represented as originating from equivalent sources at infinity.

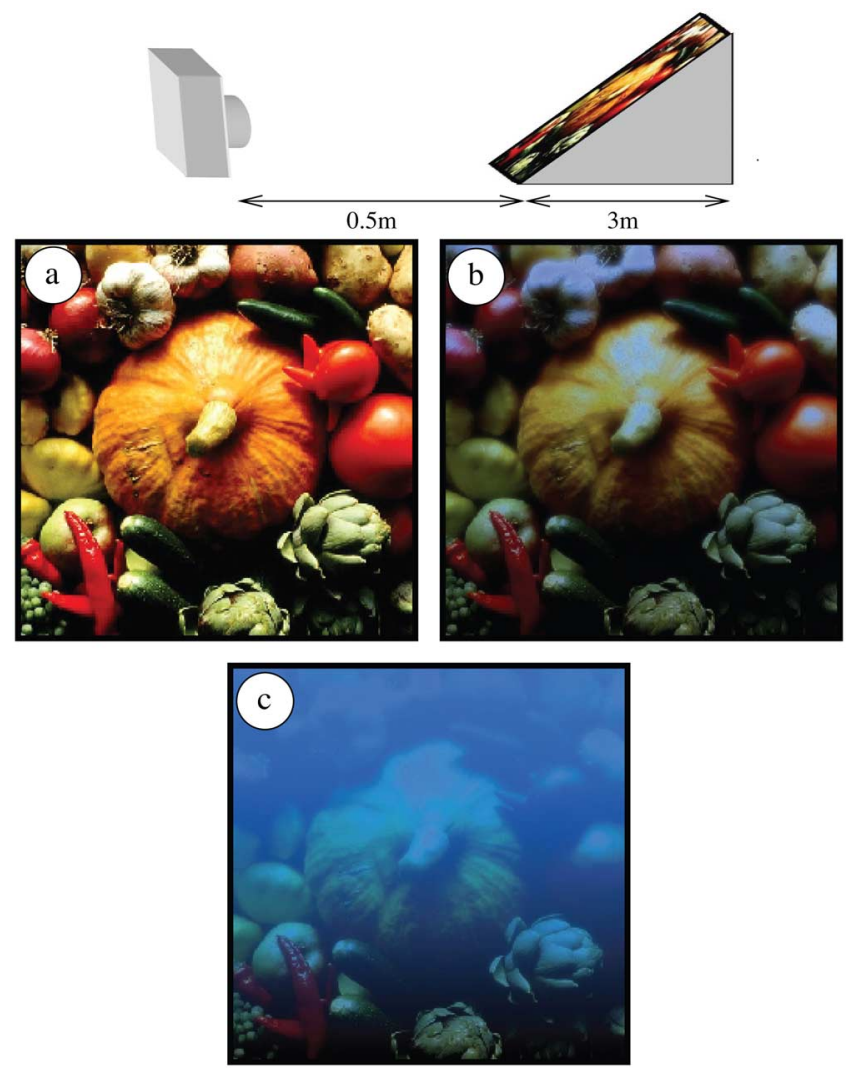

Fig. 5. Simulating underwater imaging. The bottom part of the scene is set to be $0.5 \mathrm{~m}$ away. The distance linearly changes to $3.5 \mathrm{~m}$ at the top. (a) Original image $L_{\text {object }}$. (b) Rendering the effective object $L_{\text {object }}^{\text {effective, which }}$ accounts for underwater blur (forward scattering). (c) Rendering the sensed image $I^{\text {total }}$, accounting for attenuation blur and veiling light. The latter effect is the prime cause for contrast degradation. (Color version available online at http://ieeexplore.ieee.org.)

veiling light is the dominant one. We performed a simulation of underwater imaging, whose results are shown in Fig. 5. The effects of water are simulated using a model for oceanic water [57] with a low concentration of chlorophyll and a moderate concentration of hydrosols. Fig. 5 shows a colorful set of objects with 
radiance $L_{\text {object }}$. Then it shows $L_{\text {object }}^{\text {effective }}$, which accounts for blur by forward scattering. The colors slightly change due to forward scattering since (4) includes an attenuation factor, which is implicitly wavelength dependent. We simulated the effects of varying distances by setting a distance map to the scene: the distance linearly increases from $0.5 \mathrm{~m}$ at the bottom of the image to $3.5 \mathrm{~m}$ at its top.

Incorporating veiling and attenuation effects as well as blur, the total image irradiance [29] is

$$
I^{\text {total }}=S+B=e^{-c z} L_{\text {object }}^{\text {effective }}+B .
$$

Visibility strongly deteriorates ${ }^{4}$ at image $I^{\text {total }}$. Now, even objects at moderate distances are swamped in a veiling blue light and become obscured. Veiling light affects the color and contrast of all objects.

We now turn to a quantitative analysis of the images shown in Fig. 5. As a criterion for image quality, we look at contrast. The reason is that measurement of contrast underlies the determination of the modulation transfer function (MTF) of media [64]. Moreover, contrast has been shown to correspond to underwater visual performance such as stereoscopic acuity [70]. Contrast between two points $v=1,2$ is usually defined [55], [64] as $\left|I_{1}-I_{2}\right| /\left|I_{1}+I_{2}\right|$, where $I_{v}$ is the intensity at point $v$. We generalize this definition such that in a region having $N$ pixels, we use

$$
C(I)=\frac{\operatorname{STD}\left\{I_{v}\right\}}{\frac{1}{N} \Sigma_{v=1}^{N} I_{v}},
$$

where $\operatorname{STD}\left\{I_{v}\right\}$ is the standard deviation of the $N$ intensity values. To calculate the contrast in a region of a color image having $N$ pixels, we use

$$
C(I)=\frac{\sqrt{\frac{1}{N} \sum_{v=1}^{N} \sum_{\chi=\text { red,green,blue }}\left(I_{v}^{\chi}-\bar{I}^{\chi}\right)^{2}}}{\sum_{\chi=\text { red,green,blue }} \bar{I}^{\chi}},
$$

where $\chi$ is an index of the chromatic channel and

$$
\bar{I}^{\chi}=\frac{1}{N} \sum_{v=1}^{N} I_{v}^{\chi}
$$

The contrast of the effective object $C\left(L_{\text {object }}^{\text {effective }}\right)$ and the sensed image $C\left(I^{\text {total }}\right)$ are calculated. Relative to the contrast of the original scene, the degradations of contrast are

$$
\mathcal{D}_{\text {object }}^{\text {effective }}=\frac{C\left(L_{\text {object }}^{\text {effective }}\right)}{C\left(L_{\text {object }}\right)} \quad \text { and } \quad \mathcal{D}^{\text {total }}=\frac{C\left(I^{\text {total }}\right)}{C\left(L_{\text {object }}\right)} \text {. }
$$

Before inspecting these values, we define an image resulting from all degradation effects excluding blur

$$
\tilde{I}_{\text {(no blur) }}^{\text {total }}=e^{-c z} L_{\text {object }}+B
$$

with a respective contrast degradation measure

$$
\mathcal{D}_{\text {(no blur })}^{\text {total }}=\frac{C\left(\tilde{I}_{\text {no blur }}^{\text {total }}\right)}{C\left(L_{\text {object }}\right)} \text {. }
$$

${ }^{4}$ The weight of the veiling light relative to the signal depends on the object albedo. The larger the albedo, the stronger the signal is. Based on empirical studies of typical terrestrial objects [69], we set the average albedo to 0.2 .
The contrast degradation in the scene depicted in Fig. 5 is given as a function of distance in Table I. The effect of blur alone is not negligible, especially at long distances, as reflected in the values of $\mathcal{D}_{\text {object }}^{\text {effective }}$. Nevertheless, the effect of the complementary processes is much more dramatic, as indicated by $\mathcal{D}_{\text {(no blur) }}^{\text {total }}$. While most of the contrast is maintained in $L_{\text {object }}^{\text {effective }}$, it is reduced by orders of magnitude by the veiling light, which increases while the signal is attenuated.

This observation is consistent with experiments described 5 in [64]. In addition, this observation is consistent with analogous conclusions regarding visual degradation in the atmosphere [33], [69] that ambient light scattered into the LOS is the most important contributor to aerial image degradation rather than blur. It has also been noted in psychophysical studies [71] that human perception of an "atmosphere" is attributed to the additive contribution, which is associated with the path radiance. A similar conclusion applies underwater: veiling light is the dominant contributor to image degradation.

\section{Polarization}

Underwater scattering involves polarization effects. In the following sections, these effects are exploited to compensate for underwater visibility degradation. First, however, we describe the models for these effects. Consider a narrow source that illuminates the scattering particles residing on the LOS. The narrow source and the LOS from the camera to the object define a plane of incidence. We divide the veiling light into two polarization components that are parallel and perpendicular to this plane, $B^{\|}(\boldsymbol{\Theta})$ and $B^{\perp}(\boldsymbol{\Theta})$, respectively. Typically, $B^{\perp}(\boldsymbol{\Theta})>B^{\|}(\boldsymbol{\Theta})$; i.e., light is partially polarized perpendicular to the plane.

In general, irradiance of the LOS is not due to a narrow source. Had illumination been isotropic, no specific direction in space could have set a preferred polarization vector; hence, overall the veiling light would not have been polarized. Fortunately, the LOS is generally illuminated in a highly anisotropic manner. Natural underwater light originates from the sun and sky above the water. This light is restricted to a cone around the zenith called the optical manhole [28], [72] or Snell's window [21], [40]. Scattering in the water leads to illumination of the LOS from other directions as well (e.g., from below and from the sides), but with lower intensity [47], [65]. Changes caused by this scattering to the angular irradiance distribution gradually depend on the underwater depth. As we go deeper, the illumination reaches an asymptotic distribution [43], [47], [57], [65] that is strongly peaked around the zenith.

Due to the anisotropy of the LOS irradiance, typical underwater natural veiling is significantly partially polarized [43]. As depth increases, this polarization reaches an asymptotic value, which is maintained to an infinite depth [47], [65]. The direction of polarization depends on the sun's bearing [47] (unless it is occluded by clouds) and the viewing direction. However, this orientation typically tends to be approximately horizontal [13], [21], [28], [48], [56], [58], [72], [73] for horizontal LOSs.

${ }^{5}$ In [64], the MTF was characterized using black stripes over a white paper. The albedo and contrast of that target had thus been much higher than the values we use in the simulation above, since the values we use are based on natural characteristics [69]. This leads to some moderation in [64] of the relative contribution of the veiling light to the degradation of visibility of the calibration target. Still, veiling light degraded the contrast much more than blur. 
TABLE I

CHANGES OF CONTRAST OF AN EFFECTIVE IMAGE COMPARED TO A Corresponding SEnsed BlurRed Image AND A SEnsed Image ExCluding Blur. As CAN Be SeEn, Blur is Not as Dominant as the COMPLEMENTARY EFFECTS

\begin{tabular}{c|c|c|c}
\hline$z$ (meters) & $\mathcal{D}_{\text {object }}^{\text {effective }}$ (percent) & $\mathcal{D}_{\text {(no blur) }}^{\text {total }}$ (percent) & $\mathcal{D}^{\text {total }}$ (percent) \\
\hline 0.75 & 89 & 8 & 7 \\
1.5 & 78 & 5 & 4 \\
2.0 & 73 & 3.4 & 2.7 \\
2.5 & 70 & 2.2 & 1.7 \\
3.5 & 62 & 1 & 0.7 \\
\hline
\end{tabular}

In order to sense the different polarization components, we image the scene through a polarizing filter (Fig. 2). Since natural veiling is partially polarized, then its intensity depends on the filter's orientation around the optical axis. There are two orthogonal orientations of the polarizer for which its transmittance of the veiling light reaches extremum values $B^{\max }$ and $B^{\min }$. These are the two linear polarization components of the veiling light, i.e.,

$$
B=B^{\max }+B^{\min },
$$

where $B$ is given by (13). The DOP of the veiling light is defined by

$$
p \equiv \frac{\left(B^{\max }-B^{\min }\right)}{B} .
$$

As for the signal $S$, we assume that it has insignificant influence on the polarization of the measured light relative to the influence of the veiling light. Four reasons are listed for this assumption.

1) Light reflected from rough surfaces is naturally depolarized [43].

2) Contrary to reason 1 , light reflected from specular dielectric objects may be highly polarized. However, underwater specular reflection is weaker [40] than in air since the refraction index of water is closer to that of the reflecting dielectric. As an example, Fig. 6 plots the reflectance of glass in water divided by this reflectance in air. It shows that for almost all incidence angles, the specular reflection underwater is much weaker than in air.

3) Even if light emanating from the object is partially polarized, the signal polarization decreased as the distance to the camera increases. This is caused by multiple scattering along the LOS [14], [73].

4) Even if the signal reaches the camera with substantial polarization, its influence is typically smaller than that of the veiling light. The reason is that the signal decreases (7) while the veiling light (13) increases with distance. Thus, veiling light and its polarization dominate the measurements as distance increases. Therefore, the accuracy of the model increases where it is needed most - at distant objects, which are most affected by visibility degradation.

Nevertheless, note that this assumption may not hold at very close distances in a relatively good visibility if the object strongly polarizes light as in [6], [22], and [46].

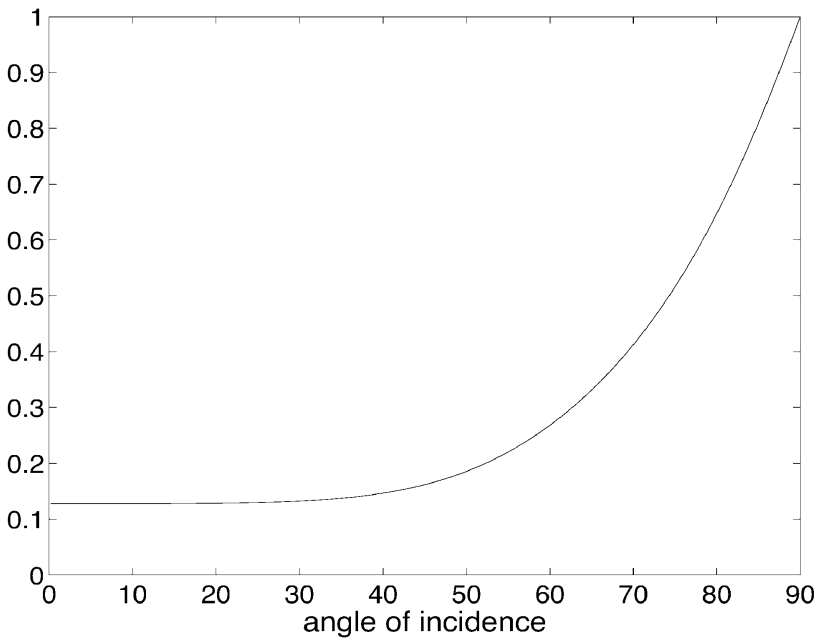

Fig. 6. Ratio of the reflectance of glass in water relative to its reflectance in air. At most angles, the values are small, indicating the weakening of specular reflectance inside the water.

\section{IMAGE ACQUISITION}

In this section, we describe the first part of our visibility restoration method: image acquisition. In particular, we describe our apparatus. When a polarizer is mounted, the sensed intensity at each image pixel changes as a function of the filter orientation angle. Similar to veiling light, there are two orthogonal polarizer angles corresponding to extremum values of intensity $I^{\max }$ and $I^{\mathrm{min}}$, where

$$
I^{\text {total }}=I^{\max }+I^{\min },
$$

while $I^{\text {total }}$ is given by (15). Since we assume that the signal polarization is insignificant, the polarizer modulates only the veiling light. This assumption is consistent with observations reported in [43] and [54]. Therefore, the raw images ${ }^{6}$ corresponding to the extrema of the intensity measure are

$$
I^{\max }=\frac{S}{2}+B^{\max } \text { and } I^{\min }=\frac{S}{2}+B^{\min } .
$$

Note that $I^{\mathrm{min}}$ is the image taken at the "best state" of the polarizer, where the disturbing veiling is minimal [48], [54]. On the other hand, $I^{\max }$ is the image taken at the "worst state" of the polarizer, where the veiling is maximal.

In order to acquire such images, we built a custom system for underwater polarimetric imaging, which we term the Aqua-Polaricam. Several specifications determined its design, as detailed in the Appendix. The system is shown in Fig. 7. The housing is manufactured by Sealux and is commercially available. For the reasons explained in the Appendix, we chose the housing with a $120-\mathrm{mm}$ dome port made of glass while an inverted circular polarizer is attached externally to it. The

\footnotetext{
${ }^{6}$ It is not necessary to use the minimum and maximum intensity values. Almost any two images (i.e., polarizer angles) that modulate the polarized component may be used. Nevertheless, the extrema images provide the strongest modulation, yielding the most reliable outcome. The exception is when the two images have identical values as occurs when the polarizer is oriented at $+45^{\circ}$ and $-45^{\circ}$ to the veiling light polarization.
} 


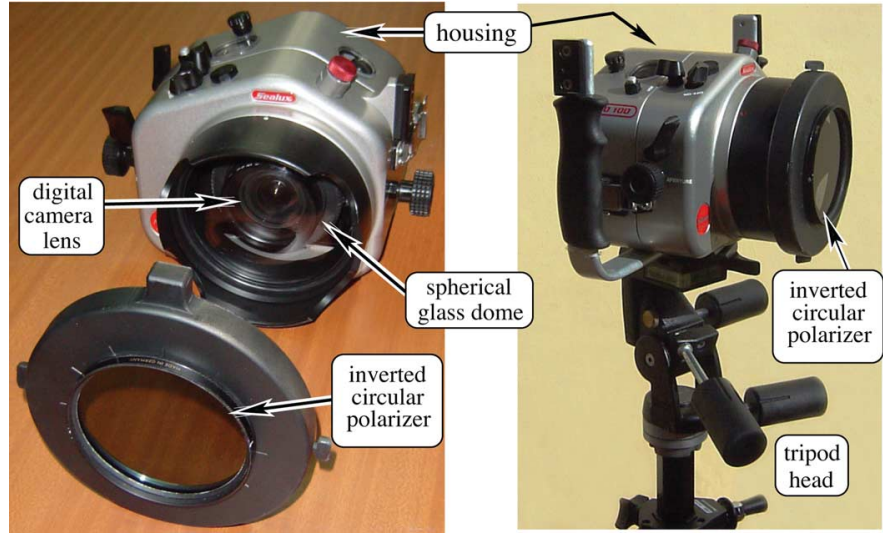

Fig. 7. The Aqua-Polaricam. (Left) With the polarizer mount separated, the dome and lens are visible. (Right) The complete imaging system mounted on a tripod. (Color version available online at http://ieeexplore.ieee.org.)

surrounding water flows to the space between the external polarizer and the dome through several openings in the housing's interface to the polarizer mount. We use the Nikon D100 digital single lens reflex (SLR) camera that enables extraction of raw output data. This data is linearly related to the scene radiance (i.e., the camera has no gamma correction in this mode) without automatic white balancing. ${ }^{7}$ We used a 20 -mm wide-angle Nikon lens. The distance $l_{o}$ to the dome was $80 \mathrm{~mm}$. Preliminary experiments revealed that stray light enters the housing from its back viewing ports and then reflects into the lens. Stray light was blocked by slightly modifying the internal structure of the commercial housing.

We performed experiments by scuba diving and taking images using the system (Fig. 8). At each scene, two photographs ${ }^{8}$ were taken to capture $I^{\max }$ and $I^{\mathrm{min}}$. As an example, we scubadived in Eilat at the Red-Sea to a depth of $26 \mathrm{~m}$ in an area containing coral reefs. The raw images ${ }^{9}$ are shown in Fig. 9. Both of the images have a very low contrast, yet their slight difference provides the key for substantial visibility improvement by a mathematical algorithm, described next.

\section{CleAR UNDERWATER ViSIBILITY}

The algorithm for improving underwater visibility overcomes the "veiling" effect caused by scattered light [48], [58]. For this reason, the adjective "unveiled" is used to describe the image resulting from the algorithm. In addition to "unveiling", we need to address the underwater illumination color bias. As we go deeper underwater, the red portion of the illumination spectrum is absorbed by the water [27]. Hence, for perceptual plausibility, we include a white balancing procedure.

${ }^{7}$ We confirmed the linear response of the system using different exposures of the MacBeth ColorChecker calibration chart.

${ }^{8}$ The method assumes that illumination does not change in time between image acquisitions, as indeed was the case in our experiments. However, due to light refraction through moving surface waves, the illumination may fluctuate. Effects arising from these fluctuations can be suppressed based on a few additional frames, as described in [74].

${ }^{9}$ For clarity of display, the brightness of the displayed pictures in this paper underwent the same standard contrast enhancement (stretching) while their hue and color saturation were untouched. The recovery algorithms, obviously, used the raw (not brightness enhanced) images.

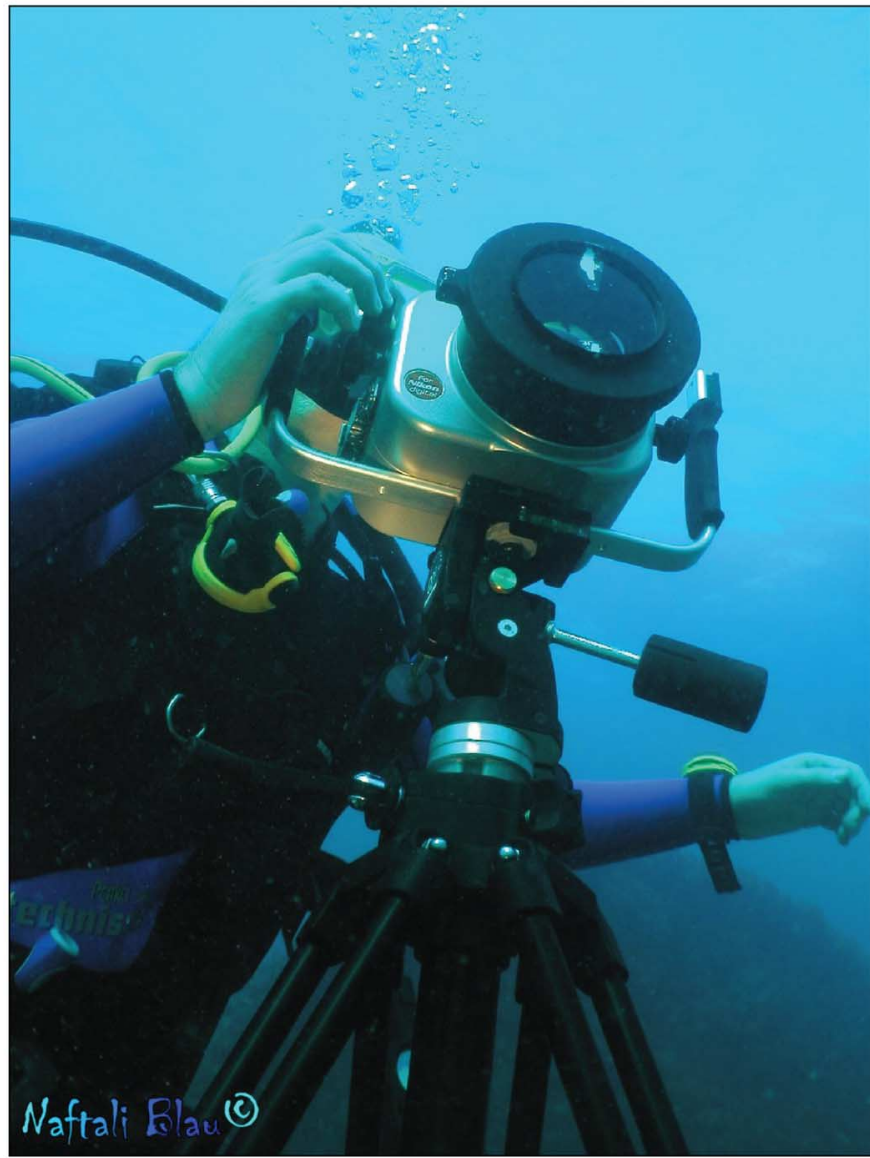

Fig. 8. Images were taken manually during scuba dives. (Color version available online at http://ieeexplore.ieee.org.)
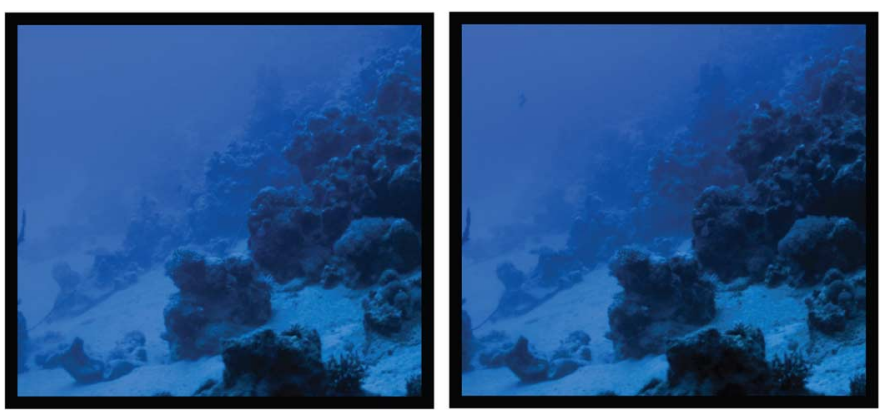

Fig. 9. Underwater scene in Eilat at the Red-Sea, $26 \mathrm{~m}$ below the water surface. The images were taken using horizontal and vertical polarizer orientations. Both images are contrast stretched, yet their visibility is poor. Their difference is hardly noticeable. (Color version available online at http://ieeexplore.ieee.org.)

\section{A. Naive Attempt for Color Correction}

As mentioned in Section I, space-invariant enhancement methods do not model the spatially varying distance dependencies of visibility problems. As a result, they are of limited utility. As an example of a naive space-invariant enhancement, let us consider a simplistic method for compensating the illumination blue color bias. We know that the sand in the diving site shown in Fig. 9 is rather white. If the scene does not include such a natural object, then a white target can be introduced to the scenery [20]. Suppose that we normalize the color of the 
best polarized raw image by the color of a sandy patch in the FOV

$$
\begin{aligned}
& I_{\text {red }}^{\text {modified }}=\frac{I_{\text {red }}^{\text {raw }}}{I_{\text {sand red }}^{\text {raw }}} \\
& I_{\text {green }}^{\text {modified }}=\frac{I_{\text {green }}^{\text {raw }}}{I_{\text {sand green }}^{\text {raw }}} \\
& I_{\text {blue }}^{\text {modified }}=\frac{I_{\text {blue }}^{\text {raw }}}{I_{\text {sand blue }}^{\text {raw }}} .
\end{aligned}
$$

This multiplicative normalization does not compensate for the additive spatially varying veiling light. For this reason, the measured color values of a white patch $I_{\text {sand red }}^{\text {raw }}, I_{\text {sand green }}^{\text {raw }}$, and $I_{\text {sand blue }}^{\text {raw }}$ depend on the image coordinates $x, y$ of the selected patch. Therefore, this compensation is ill defined.

To disambiguate the process, we measure a patch that is as close as possible to the camera. This corresponds to the bottom of the photograph. At that place, the veiling is least intense, thus the value of the white patch best represents the illumination. Nevertheless, as mentioned above, (26) does not account for the additive spatially varying veiling light. Hence, the result will not perform a proper color compensation at varying distances and will certainly not remove turbidity effects, as shown on the left part of Fig. 10.

\section{B. Recovering the Object Radiance}

We now describe the mathematical algorithm that solves the problem posed in this paper. The method inverts the visibility degradation effects and in addition enables proper compensation for the color bias. Assume for a moment that we have an estimate of the global parameters $B_{\infty}$ and $p$. From (22), (23), and (25), the veiling light is estimated as

$$
\hat{B}(x, y)=\frac{\left[I^{\max }(x, y)-I^{\min }(x, y)\right]}{p} .
$$

Inserting this estimate into (13), (15), and (24), we obtain an estimate for the "unveiled" object radiance

$$
\hat{L}_{\text {object }}^{\text {effective }}(x, y)=\frac{\left[I^{\text {total }}(x, y)-\hat{B}(x, y)\right]}{\hat{t}(x, y)},
$$

where

$$
\hat{t}(x, y)=1-\frac{\hat{B}(x, y)}{B_{\infty}}
$$

is the estimated water transmittance. The transmittance is related to the object distance $z$ by

$$
\hat{t}=e^{-c z} \text {. }
$$

Recall that all the variables in these equations are functions of the light wavelength $\lambda$. To account for the wavelength dependence, it is best to analyze the images with a fine spectral resolution. Each wavelength band can be analyzed independently. In our experiments, though, we used the traditional coarse wideband red, green, and blue (RGB) sampling of the spectrum. Therefore, the method is applied to every color channel separately.

The "unveiled" image is an estimate of $L_{\mathrm{object}}^{\text {effective. Thus, we }}$ do not compensate for image blur but only for the veiling effect

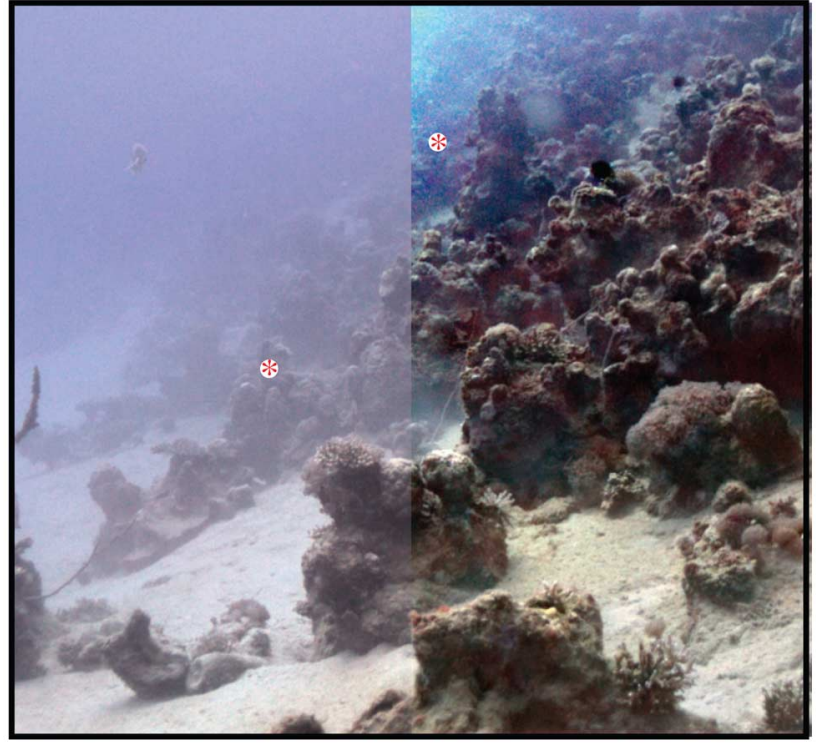

Fig. 10. Comparison between the best raw image and the recovered image These images underwent white balancing based on a close white sand patch For the raw image, this process quickly loses its effectiveness as objects become more distant. In the recovered "unveiled" image, colors are recovered to large distances. (Marked points) The regions around the points have the same contrast in their respective images. However, the point in the recovered image part is twice as distant as the one in the raw image part, indicating the increase of visibility range. (Color version available online at http://ieeexplore.ieee.org.)

of scattered light and for attenuation. At this point we make do with this estimate. The reason stems from the discussion in Section III-B: veiling light is the prime reason for image contrast degradation; hence, overcoming veiling light rather than blur is the prime step for recovering visibility.

1) Compensation for the Illumination Color: Equations (27), (28), and (29) invert the spatially varying visibility degradation effects. This enables proper compensation for the color bias of the illumination. Assume that the illumination is uniform. Similar to Section V-A, we use a patch of sand, which we know should be white, if it were not for the blue illumination color. However, contrary to Section V-A, we now perform the compensation using the recovered image $\hat{L}_{\text {object }}^{\text {effective }}$

$$
\begin{gathered}
\hat{L}_{\text {red }}^{\text {modified }}=\frac{\hat{L}_{\text {object red }}^{\text {effective }}}{\hat{L}_{\text {sand reced }}^{\text {effee }}} \\
\hat{L}_{\text {green }}^{\text {modified }}=\frac{\hat{L}_{\text {object green }}^{\text {effective }}}{\hat{L}_{\text {sand green }}^{\text {effective }}} \\
\hat{L}_{\text {blue }}^{\text {modified }}=\frac{\hat{L}_{\text {object blue }}^{\text {effective }}}{\hat{L}_{\text {sand blue }}^{\text {effective }}} .
\end{gathered}
$$

We now turn to the experiment corresponding to Fig. 9. The result of the full scene recovery algorithm (unveiling and color compensation) is shown in the right part of Fig. 10. This result can be compared to the left part of Fig. 10 in which no unveiling was attempted. Clearly, the recovered image has a much improved contrast and color. The ability to see objects in such hues under natural illumination at such an underwater depth is remarkable considering the common knowledge [27].

As another example, consider the images shown in Fig. 11. Here, the raw images were acquired at a depth of $10 \mathrm{~m}$ below 


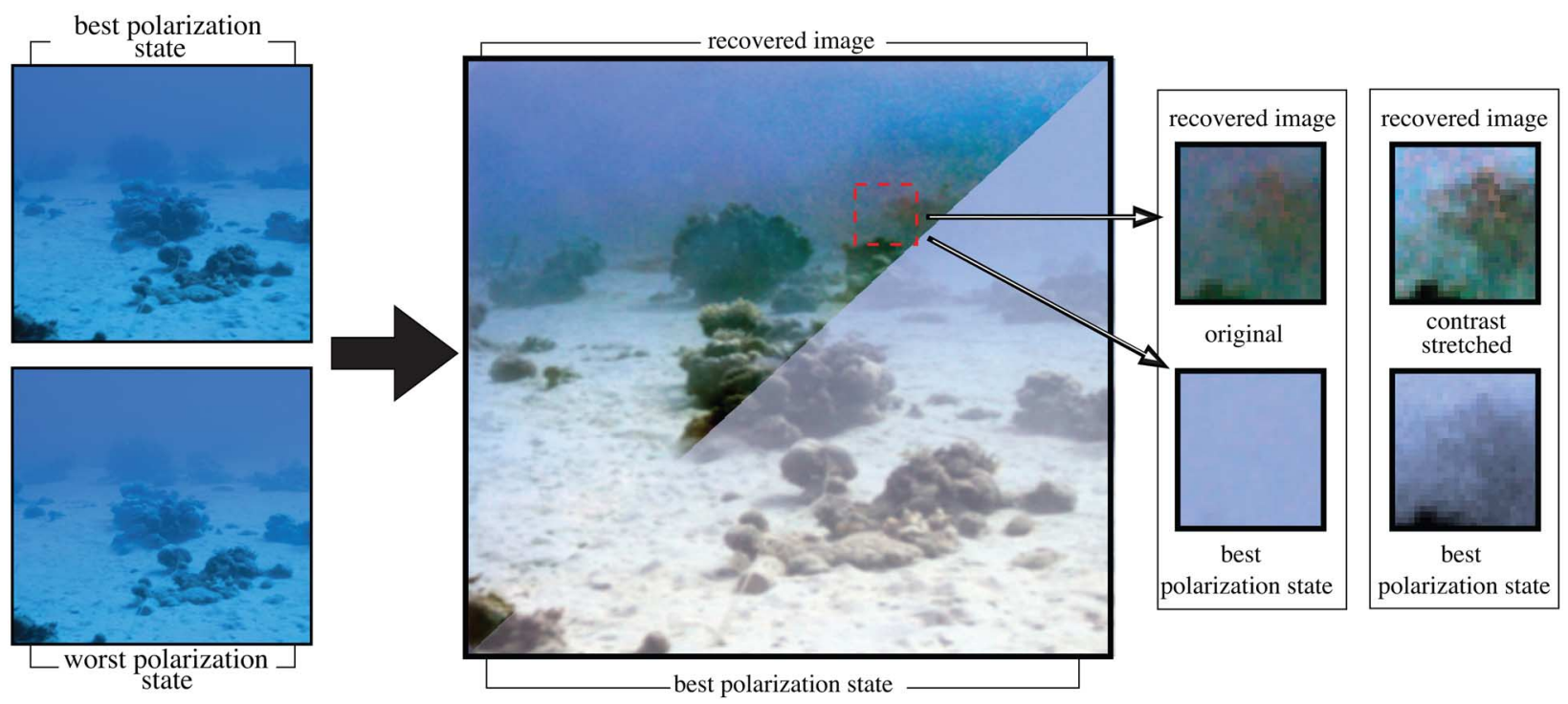

Fig. 11. Underwater scene in Eilat (the Red-Sea), $10 \mathrm{~m}$ deep. (Left) The raw images taken through a polarizer. (Middle) White-balanced results. The recovered unveiled image is much clearer, especially at distant objects, than the raw image. (Right) The recovered image reveals structures and details that are not visible in the raw image. This occurs even if a standard contrast stretch is applied to the selected area. (Color version available online at http://ieeexplore.ieee.org.)

the water surface. Similar to Fig. 10, we show the result of our unveiling algorithm in conjunction with the result obtained by a naive attempt to balance the color without unveiling. Once again, the corals in the recovered part of the picture are much clearer than in the part showing the "best polarized" image. Moreover, this recovered image reveals distant structures that are not visible in the raw images. To see this, we enlarged a square inset to the right of the figure. The enlarged square reveals a subtle structure in the recovered image, which is not visible at all in the raw image. The structure is somewhat revealed in the raw image if a standard contrast stretch is performed locally inside this square. Nevertheless, when the same contrast stretch operation is performed on the recovered image, the structure is much clearer and more detailed.

2) Quantitative Performance: Figs. 10 and 11 indicate a great deal of improvement in visual quality. However, it is interesting to seek a quantitative measure of the performance of this method and compare it to the raw image and to the result of naive color correction. We use the contrast criterion described in (17) and apply it to $I^{\text {modified }}$ defined in (26), to $\hat{L}^{\text {modified }}$ defined in (31), and to the best polarized image $I^{\mathrm{min}}$. Before applying (17), we contrast stretched the brightness of each of these images to occupy the full dynamic range $[0,1]$.

For better insight, we calculate the contrast at distinct image segments corresponding to different ranges of object distances. We obtain an estimate of these distances using the method we describe in Section VII. The results corresponding to Figs. 9 and 10 are given in Table II. The contrast of the raw image $I^{\min }$ is not monotonically decreasing with distance. The reason is that contrast depends on the scene objects whose inherent properties generally vary with their positions in the scene. Nevertheless, for each distance, the contrast resulting from naive white balancing is similar to that of the raw image. This is contrary to the improvement demonstrated in the recovered image. Similar conclusions can be drawn from Table III, which shows the results corresponding to Fig. 11.
TABLE II

RESUlts CoRRESPONDING TO THE SCENE IN Figs. 9 AND 10. The CONTRAST OF THE RAW IMAGE $I^{\text {min }}$ AND ITS WHITE BALANCED VERSION $I^{\text {modified }}$ IS COMPARED TO THAT OF THE RECOVERED IMAGE

\begin{tabular}{c|c|c|c}
\hline$c z$ & $\begin{array}{c}C\left(I^{\text {min }}\right) \\
\text { (percent) }\end{array}$ & $\begin{array}{c}C\left(I^{\text {modified }}\right) \\
\text { (percent) }\end{array}$ & $\begin{array}{c}C\left(\hat{L}^{\text {modified }}\right. \\
(\text { percent })\end{array}$ \\
\hline 0.47 & 27 & 24 & 86 \\
0.51 & 22 & 20 & 63 \\
0.91 & 8 & 8 & 53 \\
1.00 & 10 & 10 & 39 \\
1.20 & 5 & 5 & 20 \\
1.50 & 3 & 3 & 12 \\
2.30 & 2 & 3 & 9 \\
\hline
\end{tabular}

TABLE III

REsults CoRresponding to the SCENE IN Fig. 11. THE CONTRAST OF THE RAW IMAGE $I^{\text {min }}$ AND ITS WHITE BALANCED VERSION $I^{\text {modified }}$ IS COMPARED TO THAT OF THE RECOVERED IMAGE

\begin{tabular}{c|c|c|c}
\hline$c z$ & $\begin{array}{c}C\left(I^{\text {min }}\right) \\
\text { (percent) }\end{array}$ & $\begin{array}{c}C\left(I^{\text {modified }}\right) \\
\text { (percent) }\end{array}$ & $\begin{array}{c}C\left(\hat{L}^{\text {modified }}\right) \\
\text { (percent) }\end{array}$ \\
\hline 0.64 & 31 & 27 & 62 \\
0.80 & 30 & 26 & 47 \\
1.04 & 15 & 12 & 22 \\
1.24 & 5 & 3 & 13 \\
1.62 & 2 & 2 & 7 \\
\hline
\end{tabular}

\section{Sensitivity to Noise}

In this section, we analyze the sensitivity of the method to noise. Let the noise in the raw images be independent and having zero mean. Denote the STD of the noise in the raw images $I^{\text {min }}$ and $I^{\max }$ as $\sigma_{I_{\min }}$ and $\sigma_{I^{\max }}$, respectively. The noise variance of the unveiled image is

$$
\sigma_{\text {recovered }}^{2}=\left(\frac{\partial \hat{L}_{\text {object }}^{\text {effective }}}{\partial I^{\min }}\right)^{2} \sigma_{I^{\min }}^{2}+\left(\frac{\partial \hat{L}_{\text {object }}^{\text {effective }}}{\partial I^{\text {max }}}\right)^{2} \sigma_{I^{\max }}^{2} .
$$


Differentiating (28) and making use of (27) and (29)

$$
\begin{aligned}
& \frac{\partial \hat{L}_{\text {object }}^{\text {effective }}}{\partial I^{\max }}=\frac{1}{\hat{t}^{2}}\left[\left(1-\frac{1}{p}\right) \hat{t}+\frac{\hat{L}_{\text {object }}^{\text {effective }} \hat{t}}{p B_{\infty}}\right], \\
& \frac{\partial \hat{L}_{\text {object }}^{\text {effective }}}{\partial I^{\text {min }}}=\frac{1}{\hat{t}^{2}}\left[\left(1+\frac{1}{p}\right) \hat{t}-\frac{\hat{L}_{\text {object }}^{\text {effective }} \hat{t}}{p B_{\infty}}\right] .
\end{aligned}
$$

Consider the case where system noise is roughly determined by system quantization of the image irradiance to $n$ bits. Then the noise STD of the raw images is equal to

$$
\sigma_{I^{\min }}=\sigma_{I^{\max }}=\sigma=2^{-n}
$$

where the dynamic range of the imaging system is normalized to 1. Inserting (33), (34), and (35) into (32), the STD of the recovered image is

$$
\sigma_{\text {recovered }}=\frac{\sigma \sqrt{2}}{\hat{t}} \sqrt{1+\frac{1}{p^{2}}\left(1-\frac{\hat{L}_{\text {object }}^{\text {effective }}}{B_{\infty}}\right)^{2}} .
$$

This noise depends on the recovered scene radiance $\hat{L}_{\text {object }}^{\text {effective }}$, thus somewhat complicating its analysis. Nevertheless, interesting insights can be drawn from this result. First, note that if the polarization of the veiling light is very low, i.e., $p \rightarrow 0$, then the recovery is very unstable. This is understandable since low polarization does not convey significant information beyond a simple unpolarized image and hence is not useful for reliable recovery. To contain the output noise (36), we may thus need to use in the recovery equations (27), (28), and (29) a value of $p$ that is slightly biased relative to the true one. Moreover, instability always occurs at pixels that correspond to large distances, where $\hat{t} \rightarrow 0$, since there the signal $S$ may be lower than the system noise. Hence, $\hat{t}$ may need to be biased as well. These ideas are further explored in Section V-D.

Accounting for the distance dependence of $\hat{t}$ in (30) and the dependence of noise on quantization bits [see (35)]

$$
\sigma_{\text {recovered }} \propto e^{c z-n \ln 2} .
$$

The signal-to-noise ratio (SNR) decreases exponentially with the distance $z$. This is not a distinctive shortcoming of our polarization-based method. Rather, it is due to the attenuation of the signal with the distance from the object, as is the case for other underwater imaging modalities. In methods based on artificial illumination, the SNR decreases even faster as a function of object distance. The reason is that radiation is attenuated for approximately twice the object distance, beyond the $1 / z^{2}$ falloff of the artificial illumination incident on the object, caused by free space propagation. Equation (37) indicates that for a given level of output noise, the visibility range is proportional to the number of reliable camera bits. Thus, information from a 12-bit camera can recover objects that are $50 \%$ farther than those recoverable from data taken by an 8-bit camera if quantization is an effective indication for the overall noise.
Noise can be reduced by spatial filtering, e.g., local averaging. Performing this directly on the raw images or on $\hat{L}_{\text {object }}^{\text {effective }}(x, y)$ would result in image blur; however, there is a way to bypass this problem. Note that $\hat{B}(x, y)$ and $\hat{t}(x, y)$ do not vary rapidly across the FOV. The veiling light is typically a smooth function of spatial location, contrary to the rapid spatial variations of intensity and texture of the object $L_{\text {object }}(x, y)$. Thus, spatial noise filtering of $\hat{B}(x, y)$ and $\hat{t}(x, y)$ does not blur $\hat{L}_{\text {object }}^{\text {effective }}(x, y)$, yet it decreases the noise in $\hat{L}_{\text {object }}^{\text {effective }}(x, y)$.

\section{Estimation of $B_{\infty}$ and $p$}

To perform scene recovery, estimates of the global parameters $B_{\infty}$ and $p$ are needed. These are intrinsic parameters of the water and lighting. This estimation is similar to algorithms developed for open-air imaging [32]. These estimates are obtained by measuring pixels corresponding to the water background, i.e., of objects so distant inside the water that their signals are negligible due to attenuation. ${ }^{10}$ Let us denote these measured values by $\hat{p}$ and $\hat{B}_{\infty}$.

The value $\hat{p}$ obtained from direct measurement of image pixels should be slightly modified for two reasons. The first is to limit instability, as described in Section V-C. The second is the need to minimize the instances of negative pixel values. Negative pixel values stem from inaccurate measurement of $\hat{p}$ and $\hat{B}_{\infty}$ and from image noise. From (15) and (27)

$$
\hat{S}(x, y)=\frac{\hat{I}^{\text {total }}(x, y)}{\hat{p}}[\hat{p}-\hat{P}(x, y)]
$$

where

$$
\hat{P}(x, y)=\frac{I^{\max }(x, y)-I^{\min }(x, y)}{\hat{I}^{\text {total }}(x, y)}
$$

is the DOP of the measured scene (i.e., the signal combined with the veiling light). If our estimate of the veiling light DOP is too low $(\hat{p}<p)$, then negative values can appear in the image of the signal. This is especially relevant to distant objects because $P(x, y) \rightarrow p$ when $z \rightarrow \infty$.

Both of these problems can be considerably alleviated with ease if we slightly bias $\hat{p}$ by multiplying it by a factor $\epsilon$ such that $1 \leq \epsilon \leq 1 / \hat{p}$

$$
\hat{p} \rightarrow \epsilon \hat{p}
$$

The increased $\hat{p}$ makes it less likely to encounter negative values in (38). In addition, noise amplification becomes bearable. While it is clear from (36) that using a larger $\hat{p}$ decreases the noise, this is not the major contribution of this move since we advocate using only a small bias. Rather, conditioning is obtained mainly thanks to the influence of this step on $\hat{t}$. As seen from (27) and (29), increasing $p$ leads to an increase of $\hat{t}$. This eliminates the instances of $\hat{t} \rightarrow 0$ in (36). The noise in pixels corresponding to distant objects is then

$$
\sigma_{\text {recovered }}(z=\infty)=\sqrt{2} \sigma\left(1-\frac{1}{\epsilon}\right)^{-1} .
$$

\footnotetext{
${ }^{10}$ The visibility range underwater is very short. Therefore, there are usually plenty of horizontal viewing directions in which no object is visible.
} 


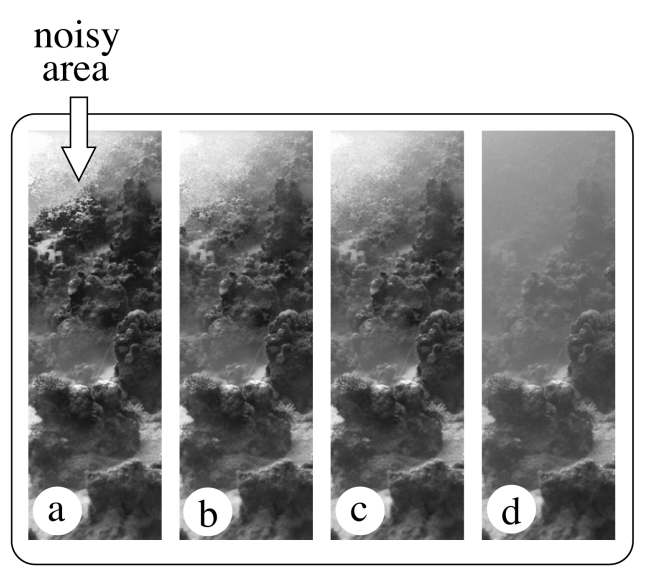

Fig. 12. Results of the recovery based on different values of the bias factor $\epsilon$. (a) No bias, $\epsilon=1$. The image is noisy at pixels corresponding to distant objects. (b) $\epsilon=1.05$. (c) $\epsilon=1.13$ : minor bias. These results look similar to (a), yet with lower noise. (d) Recovery using $\epsilon \hat{p}=1$ is equivalent to the raw image.

Thus, the unveiling process amplifies noise in pixels corresponding to distant objects, but this amplification decreases with the increase of $\epsilon$.

How does this bias affect the recovered intensity and color? It can be shown that when $\epsilon>1$, the estimated value of objects at infinity is

$$
\hat{L}_{\text {object }}^{\text {effective }}(z=\infty)=B_{\infty} \text {. }
$$

Thus, intensity and color of the raw unpolarized image are automatically retained in the recovered pixels at infinite distance. Thus, the result will have a bluish background, as is perceptually expected from an underwater image. In addition, the original raw value is retained at $z=0$ where

$$
\hat{L}_{\text {object }}^{\text {effective }}(x, y)=\hat{I}^{\text {total }}(x, y) \text {. }
$$

At all other distances, the inverse solution (28) is moderated by weighting it with the "best polarized" raw image. By using a small value of $\epsilon>1$, the result is very similar to (28) but stabilized at the problematic pixels.

These aspects are demonstrated in Fig. 12, showing results on part of the FOV of the scene shown in Fig. 9. On one extreme, Fig. 12(a) shows that when no bias is used $(\epsilon=1)$, there is excessive noise in an area corresponding to a long distance. On the other extreme, Fig. 12(d) shows the result of maximum bias, i.e., $\epsilon=(1 / \hat{p}) \approx 8$. Here, the result is simply the "best raw" image after undergoing the simple white balancing operation described in Section V-A. As these are the extreme cases, their difference is significant. The situation is, however, different in Fig. 12(b) and (c) for which regularization is minor: $\epsilon=1.05,1.13$. These results look very similar to the one corresponding to $\epsilon=1$, except for distant objects. At long distances, noise is effectively reduced to a tolerable level.

Conveniently, the results were not very sensitive to the precise value of $\epsilon$, as seen from the comparison of Fig. 12(b) and (c). This parameter does, however, offer the user a degree of freedom in deciding how aggressive the scene recovery should be. This is analogous to parameters used in standard regularization techniques, where typically a user-selected parameter

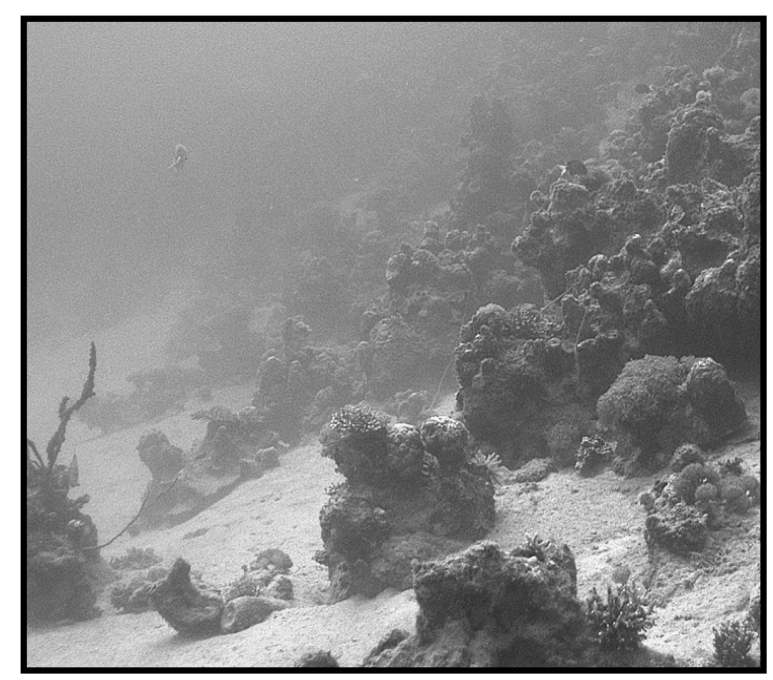

Fig. 13. Image after unsharp masking. Noise is somewhat amplified. A color version of this image can be found at [76].

trades a data-fitting term against an image-smoothness term. A benefit of this simple technique is that it does not impose blur for noise control, contrary to standard regularization. However, we do not claim this to be the optimal method for limiting noise amplification. We currently pursue further development of this issue. In the experimental results shown in Figs. 10 and 11, we used $\epsilon=1.13$.

\section{COMPARISON TO PRIOR METHODS}

In this section, we compare our results to prior methods that rely on natural illumination (see Section II). First, we discuss the standard image processing procedures of histogram equalization [75] and unsharp masking. Then, we discuss prior polarization-based methods.

Both histogram equalization and unsharp masking are spaceinvariant methods and are therefore useful when contrast degradation is uniform across the raw image. As previously described, underwater the contrast loss is strongly affected by the distance and is therefore spatially variant. As shown here, the effectiveness of the standard image processing method is limited. Unsharp masking [75] is a linear enhancement method in which the image Laplacian is added to the original image. In Fig. 13, this method sharpens the image, but only slightly improves the long-range visibility, while increasing noise. It is noted that unsharp masking can be applied to our recovered image should we want to sharpen it in trade of noise.

In Fig. 14, histogram equalization of the raw image slightly improves the areas corresponding to distant objects, but on the other hand the visibility of close objects is worse (saturated). Furthermore, because the histogram of an image depends on the objects in the scene, the histogram of a partial frame image is different than that of the whole frame, leading to inconsistent results (Fig. 14). Moreover, histogram equalization of color images is ill defined, and therefore colors are generally distorted by this operation. Hence, the histogram-equalized image is displayed in grayscale.

The DOP [5] and polarization difference imaging (PDI) methods [2], [39] are based on imaging through a polarizer. 

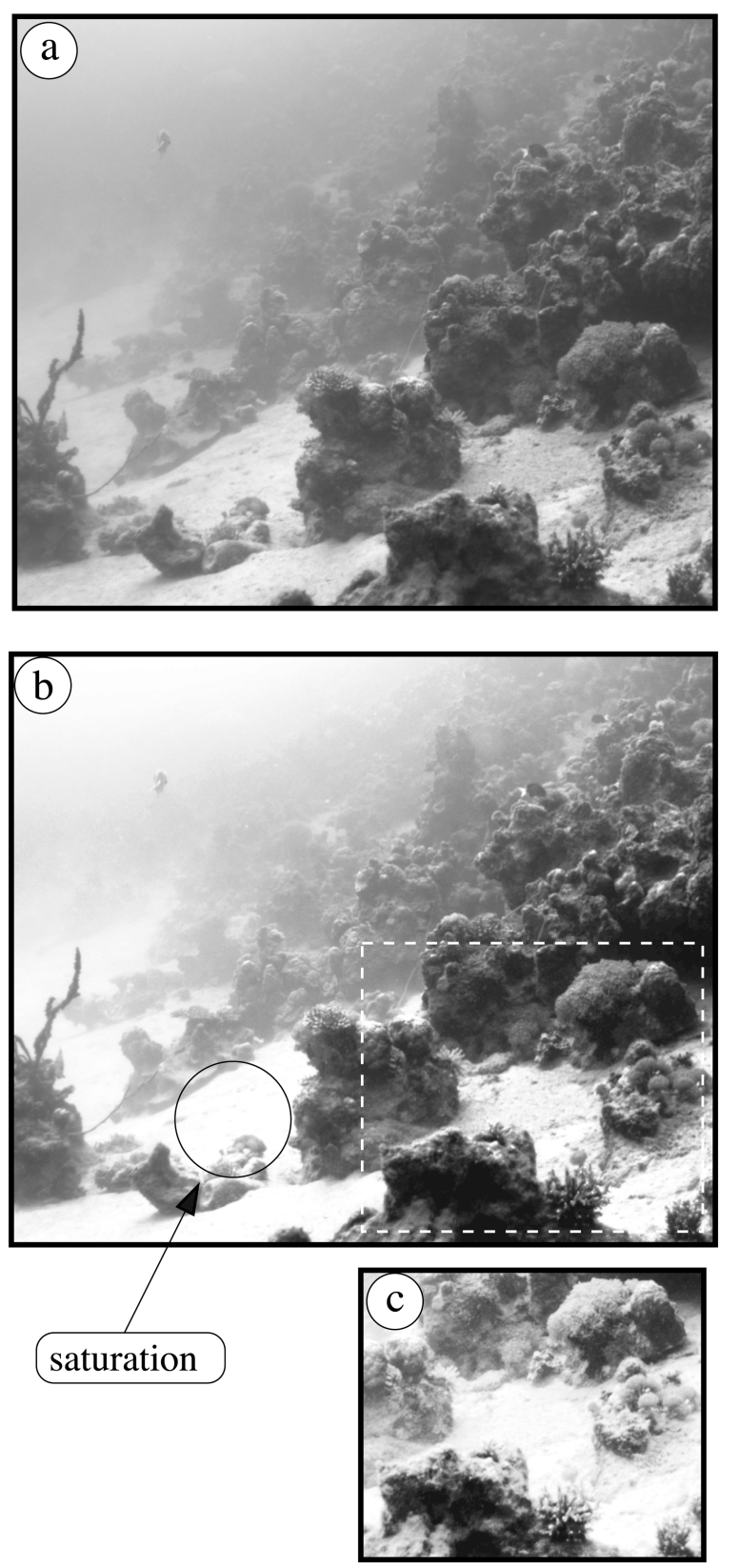

Fig. 14. (a) Image $I^{\mathrm{min}}$ in grayscale. (b) Image after histogram equalization. While visibility is somewhat improved in some areas, it is worse in others. As seen when comparing with (c), histogram equalization is inconsistent when applied to part of the frame.

DOP and DPI use $I^{\max }$ and $I^{\min }$ differently than our method and implicitly assume that polarization is associated with the signal rather than the veiling light, contrary to our method. Fig. 15 shows the results of those methods as applied to the raw data shown in Figs. 9 and 11. Clearly, the prior methods do not recover the scenes at all, especially at long distances. ${ }^{11}$ Recently, Chang et al. [58] suggested a different equation to enhance underwater polarization images. That method is

\footnotetext{
${ }^{11}$ It is interesting to observe in Fig. 15 that the DOP is high in some pixels corresponding to corals at close distances. The reason is that some points on the corals are very dark, thus light measured in these points is dominated by veiling light. Therefore, their polarization is significant.
}

formulated for grayscale images. The results of this formation are given in Fig. 16 and indicate that it has been unsuccessful in our experiment.

\section{How FAR DO WE SEE?}

As a by-product of the radiance recovery process, we get an estimate of the distance map of the scene. From (30), the distance $z$ is estimated as a function of $(x, y)$ up to a global scale factor $c$. It is given by

$$
\widehat{c z}(x, y)=-\ln \left[1-\frac{\hat{B}(x, y)}{B_{\infty}}\right] .
$$

Unless a transmissiometer is used, we do not know what the attenuation coefficient $c$ is. Nevertheless, we can quantitatively determine the relative distances in the scene. Note that if we know the distance to a single point, we can set the scale of the relative distance map. We can then derive the absolute distance to any point in the scene as well as the attenuation coefficient.

Similar to Section V-C, analyzing the noise sensitivity yields the noise STD of the recovered distance as

$$
\sigma_{\hat{z}}=\frac{\sigma \sqrt{2}}{c p B_{\infty}} e^{c z}
$$

Equation (45) indicates that this noise is greatly amplified if $B_{\infty} \rightarrow 0$. Recall that the illumination of scenes at deeper water typically suffers from low energy in the red portion of the spectrum, thus $B_{\infty}^{\text {red }} \ll B_{\infty}^{\text {blue }}$. Hence, the distance recovery at the blue portion of the spectrum can be expected to be more reliable. Therefore, in our experiments, we derived the relative distance maps using (44) based on the blue channel. These maps are shown in Figs. 17 and 18.

Thanks to the relative distance map, we can compare two image regions and determine that one of them is, say, three times as distant from the camera as the other one. This fact enables assessing the ratio of improvement of the visibility range achieved by the recovery method. The visibility range is the distance at which we may still observe certain details [77], [78]. Thus, to calculate the ratio of visibility ranges, we should compare the appearance of the same object at different distances. We have yet to conduct controlled experiments using standard targets. Nevertheless, for a rough estimate, we selected from the scene, appearing in Fig. 9, two regions that have the following characteristics.

1) Both regions have a similar object content.

2) The contrast level of one region in the raw image matches the contrast of the second region in the recovered image.

The selected pair of regions is around the marked points in Fig. 10. Both regions contain the same type of objects: chunks of the coral reef. We therefore assume that the intrinsic object properties are the same in these two regions. The contrast $C\left(I^{\text {modified }}\right)$ of the marked left region in the raw image is the same as the contrast $C\left(\hat{L}^{\text {modified }}\right)$ of the marked right region in 

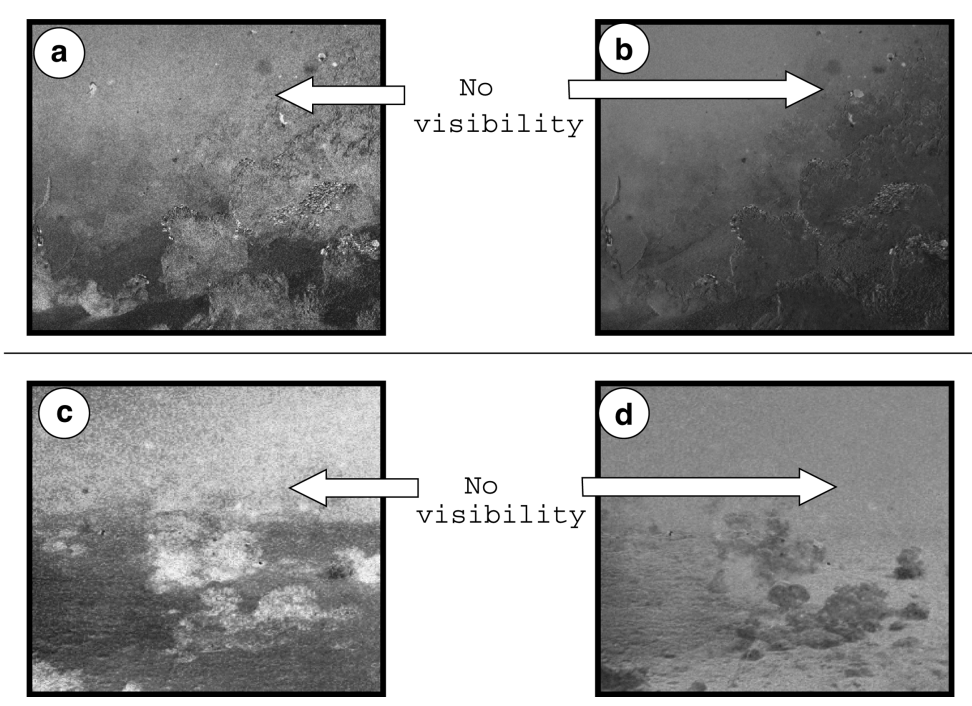

Fig. 15. Results of past methods for polarization-based enhancement. (a) DOP and (b) PDI, as applied to the images shown in Fig. 9. (c) DOP and (d) PDI, as applied to the images shown in Fig. 11. Those methods yield poor results. Visibility at long distances is not enhanced.
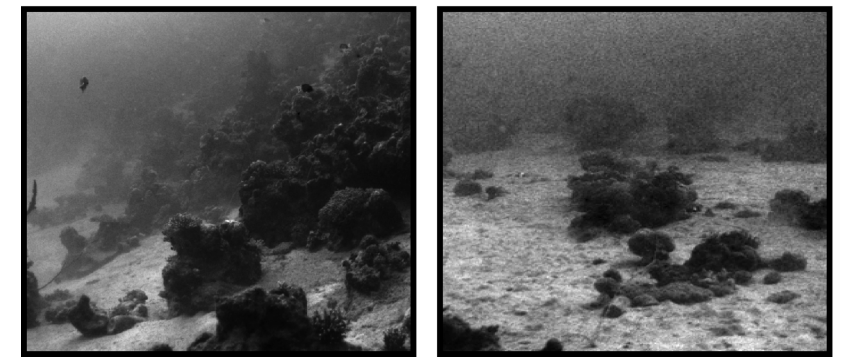

Fig. 16. Results of the method suggested in [58]. No significant improvement is noticed at long distances. Compare to the color images in Figs. 10 and 11.

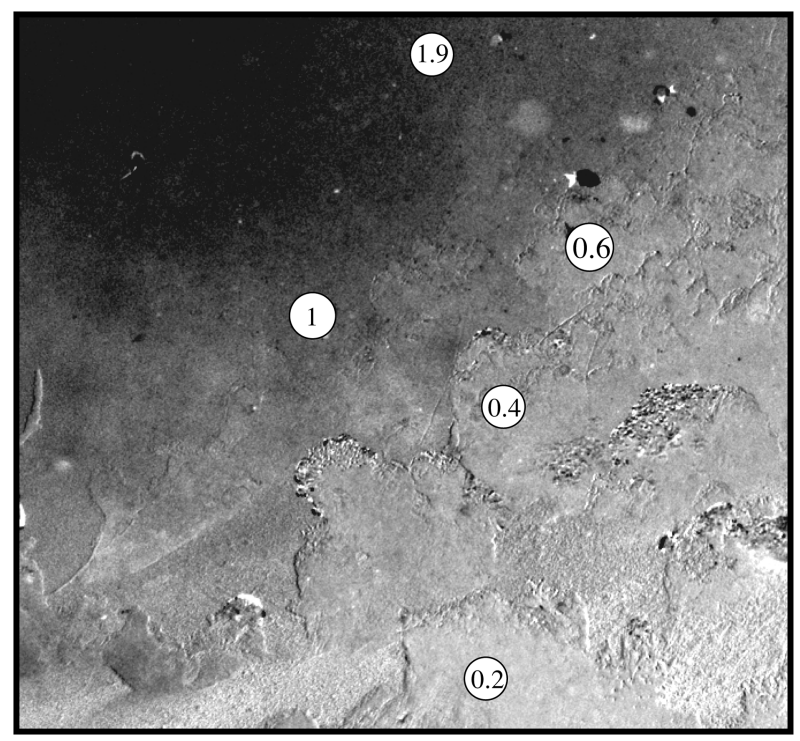

Fig. 17. Estimated range map of the object in Fig. 10. Longer distances are displayed by darker gray levels. The distance to some points is written in units of the attenuation distance $c^{-1}$ of the blue channel.

the recovered image. ${ }^{12}$ To conclude, both regions have a similar object content. The contrast level of one region at a certain dis-

\footnotetext{
${ }^{12}$ In order to minimize the contribution of noise, we estimated $C$ using only the blue channel in (16).
}

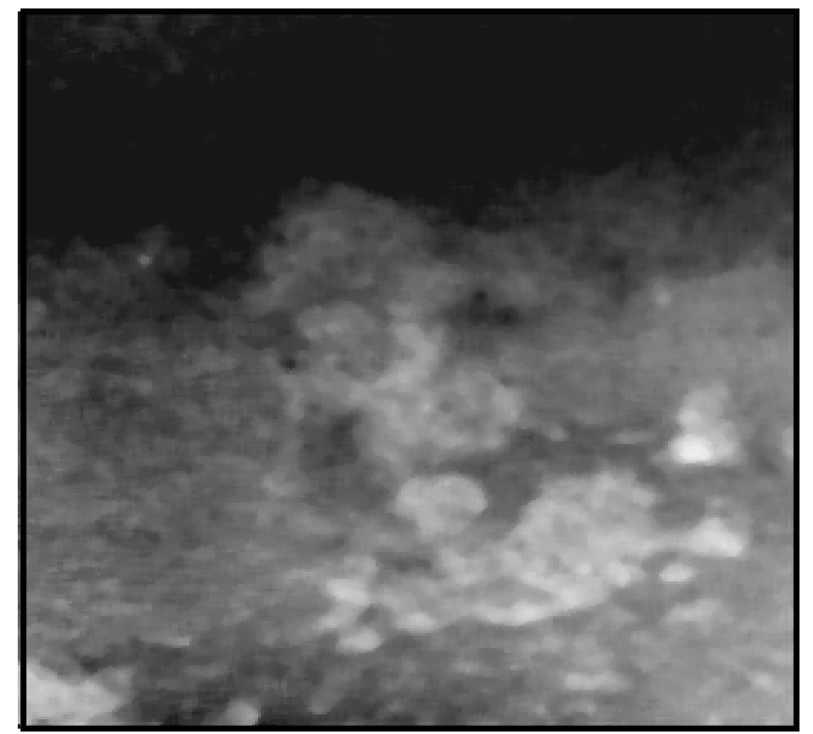

Fig. 18. Estimated range map of the object in Fig. 11. Longer distances are displayed by darker gray levels.

tance in the raw image matches the contrast of the second region in the recovered image but at a longer distance. Using (44), the range ratio between the points is 1.8 . We therefore conclude that the method demonstrated an increase of the visibility range by a factor of $\approx 1.8$. We plan to enhance the credibility of this estimate in the future.

The recovered range map can be used to render the scene from viewpoints other than the ones used during acquisition. We used the estimated range map to create a three-dimensional (3-D) surface and changed the viewpoint. To emphasize the difference between the viewpoints, we inserted virtual objects into the scene (four spheres) as in Fig. 19. Note the occlusions due to the terrain. This visual effect is clearly seen in a movie that can be downloaded at [76]. For example, one of the spheres is completely seen in Fig. 19(a), where in Fig. 19(d) it is mostly occluded. Figs. 19(b), (c), and (d) are rotated by $13^{\circ}, 19^{\circ}$, and $25^{\circ}$ relative to the normal viewing direction of Fig. 19(a). See [76] for better effects. 


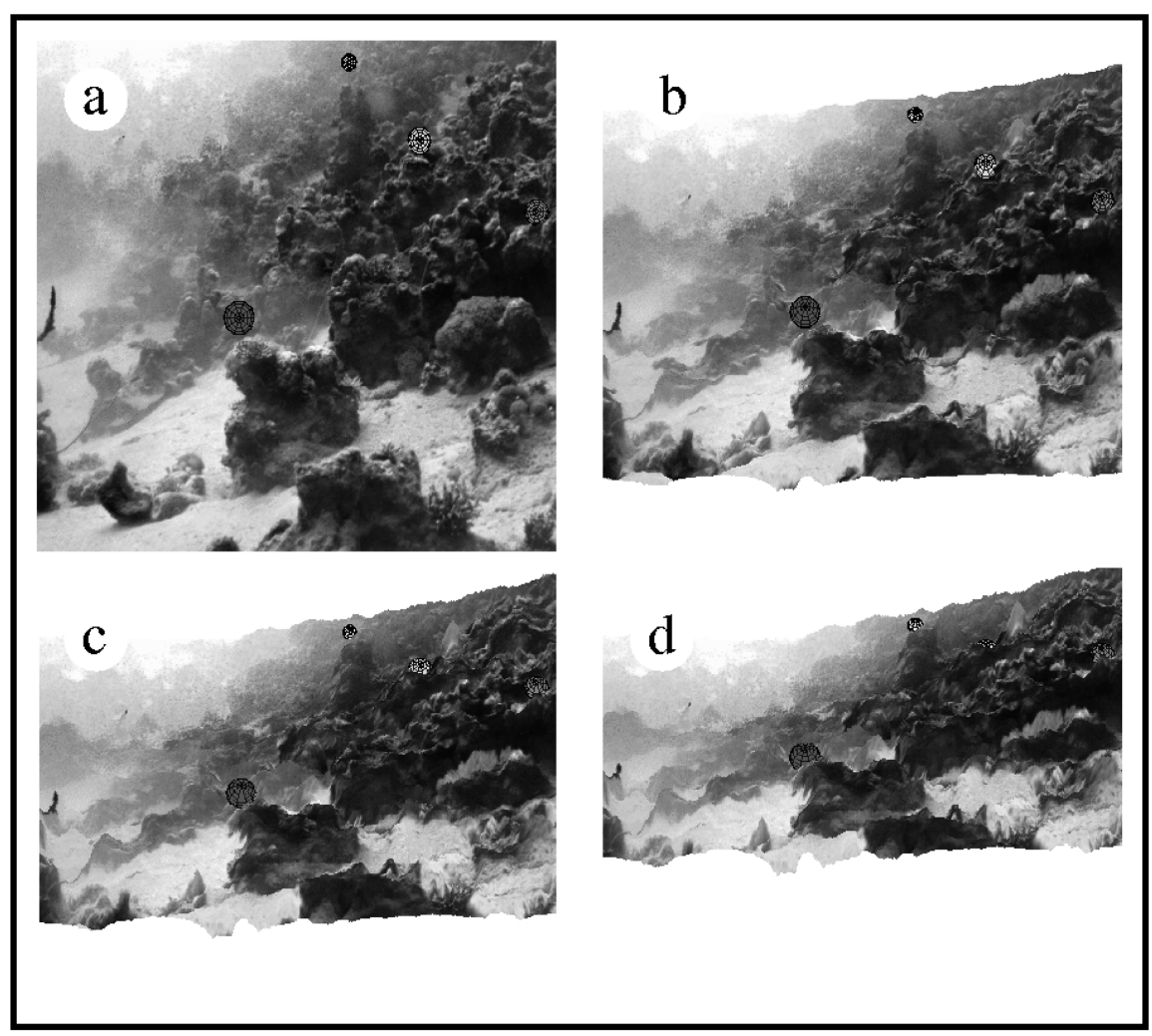

Fig. 19. We use the recovered image (a) together with the recovered range map described in Section VII to render 3-D images of the scene as if seen from different viewpoints. In addition, we inserted virtual objects (spheres) into the 3-D scene to illustrate occlusion effects. (b), (c), and (d) are arbitrarily rotated by angles of $13^{\circ}, 19^{\circ}$, and $25^{\circ}$ relative to the normal viewing direction presented in (a).

\section{SUMMARY OF THE METHOD}

For convenience, we hereby summarize our method.

1) Acquire two images through a polarizing filter, at two different polarizer angles, preferably corresponding to extremum intensity values.

2) Select an area in the image where only the water background is seen. From this area, measure $\hat{p}$ and $\hat{B}_{\infty}$.

3) Apply (40) in order to enhance stability, as described in Section V-C.

4) Apply (27) to recover the veiling light $\hat{B}(x, y)$. Optionally, apply spatial noise filtering to $\hat{B}(x, y)$, as described in Section V-C.

5) Apply (44) to obtain a distance map. Optionally, prefer a color channel that yields better stability, as described in Section VII.

6) Apply (24), (28), (29), and (31) to obtain the recovered scene.

\section{DISCUSSION}

We presented a method that can overcome degradation effects occurring in underwater vision. The method is based on a simple analysis of a pair of images acquired through a polarizing filter. Since it is physics based, the method also recovers information about the scene structure (distances). The algorithm does not require any calibration of the environmental parameters and exploits natural lighting. We believe that this approach can lead to useful tools in underwater photography, underwater research, and underwater technological applications.
The practical ability to see farther into the water with high clarity may change some of the common practices of underwater photography. Underwater photography has focused on very short distances using wide-angle lenses or macrolenses. However, the practical ability to see farther through water turbidity enables zooming on distant objects. This aspect is particularly important in marine biology research, where close encounters may affect animal behavior.

The current formulation of the method has limitations. It assumes uniform lighting along the LOS and is thus best compatible with horizontal photography rather than acquisitions in the downwards direction. In addition, the uniform lighting assumption is invalid if artificial sources are used, as is the case in deep-water explorations. Generalization of our approach to such cases is a subject for further research. An additional challenge is to extend the method to handle polarizing objects at close distances. In addition, we are planning to conduct some experiments with standard targets in order to quantitatively validate the recovery.

\section{APPENDIX \\ BUILDING AN UNDERWATER POLARICAM}

As mentioned in Section IV, several considerations have dictated the configuration of our underwater imaging system. They arise since we make quantitative photometric measurements with the polarizer. In this Appendix, we detail those considerations. This will enable the reproduction of our results 


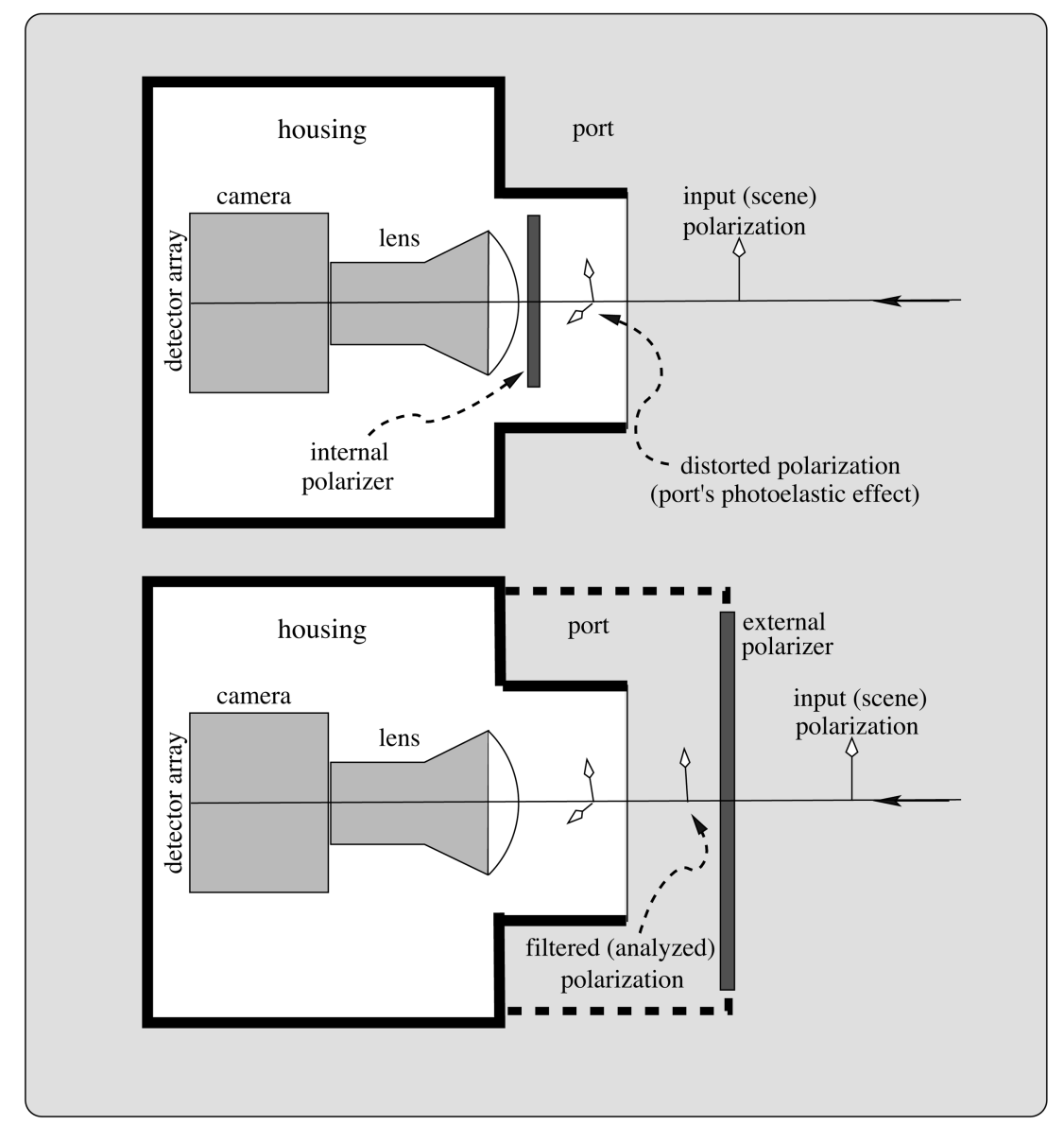

Fig. 20. (Top) Unwanted effect: photoelasticity in the port (window) changes the polarization. When light passes through an internal polarizer, this manifests in changes of image intensity and color. (Bottom) The preferred design: placing the polarizer externally to the port minimizes this effect.

by others and indicate important aspects that may be faced in future developments of this imaging approach.

For reduced noise in the scene radiance measurements, the camera should have a linear radiometric response, besides being of low noise. For flexibility in this research phase, we required full control of the camera parameters (exposure time, aperture, etc.) while it is placed in a watertight housing. The most interesting aspects of the system are related to its optical properties, as described next.

\section{Optical Considerations}

The main concern in the optical design is its effect on polarization. We use a polarizer to analyze the scene. However, we would like the rest of the optical system components to have minimal effects or sensitivities related to polarization. We achieve this by making the following decisions.

An external or internal polarizer? Stress in the transparent port's material changes the polarization of the light it transmits. This phenomenon is called the photoelastic effect [79]. Due to inhomogeneities in the material, this polarization effect is spatially varying. In our case, if the polarizer was inserted in the housing (Fig. 20), this could spatially vary the transmittance though the polarizer, depending on $\lambda$ and the polarization state. Moreover, the effect may vary with the underwater depth due to changes in the external water pressures.
In principle, placing the polarizer externally should eliminate visible photoelastic effects. We thus decided to place the polarizing filter outside the housing. Consider the bottom part of Fig. 20. The filter is the first optical component the light from the scene encounters as it enters the imaging system. The space between the external polarizer and the port is filled with the water coming from the surroundings. In practice, the photoelastic visible effects are indeed greatly diminished. However, we were concerned that residual effects persist (as has been suspected in [54] with regard to birefringence in diving masks) due to complicated refractions in the transparent material. To minimize such residual effects, we made the following decision.

A glass window. The photoelastic effect is much smaller in glass than in polycarbonate (plastics) materials [79]. We thus decided to use a glass port. We avoid the use of crystal glass windows, which are commercially available, since they may posses birefringence, possibly affecting the polarization readout.

A dome port or a flat port? Consider Fig. 21. The chief ray from an object point in the water to the detector undergoes an angular deviation [26], [27] at flat window interfaces. In this case, window transmittance depends on the polarization of the passing light [79] as can be derived from the Fresnel equations (Fig. 22). This polarization dependence distorts the intensity readouts and therefore affects the polarization estimation.

Dome ports, on the other hand, alleviate most of this problem. If the dome's center coincides with the center of projection of the camera, then the chief ray from an object point to the de- 


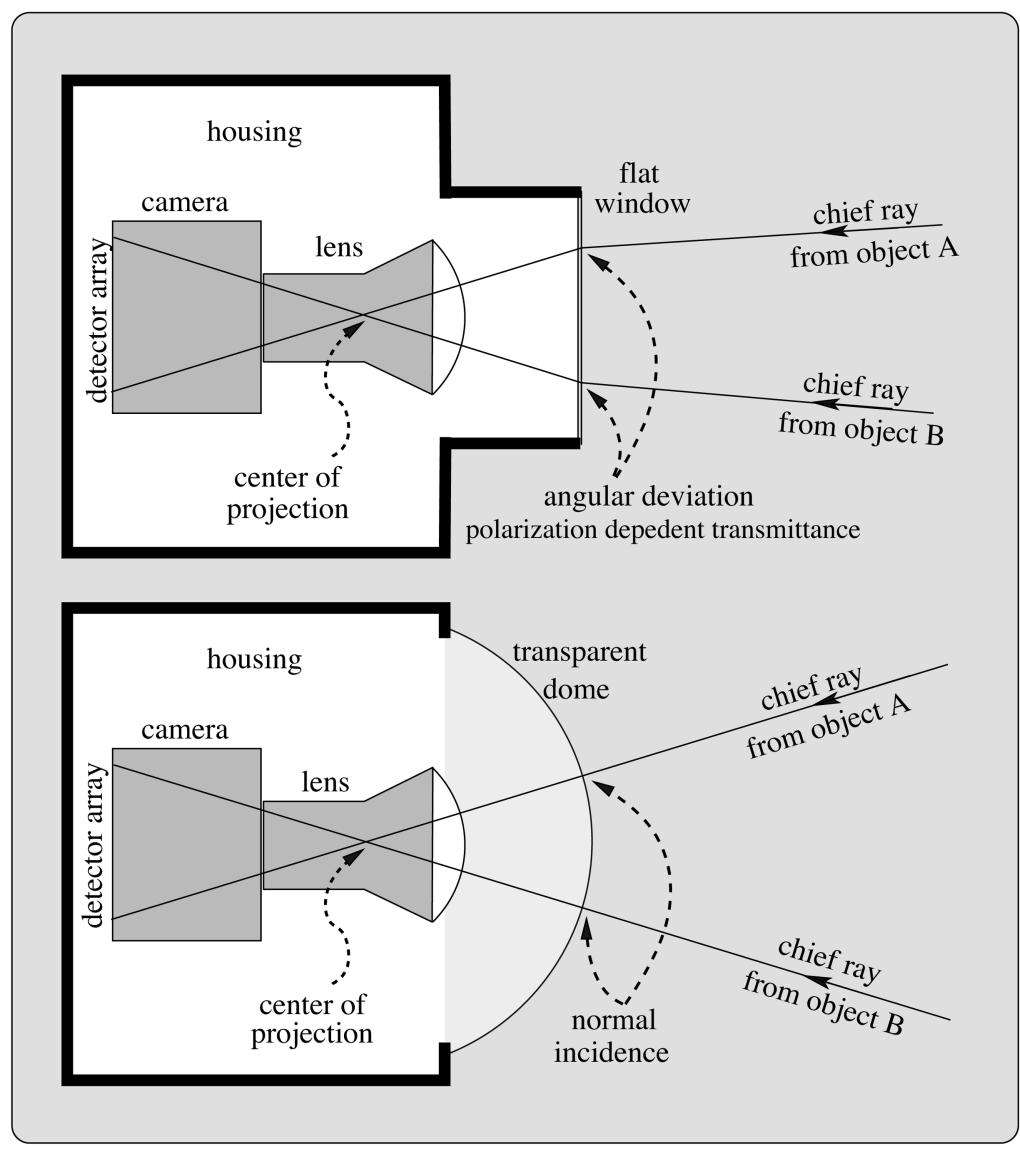

Fig. 21. (Top) Unwanted effect: the transmittance of a flat port is polarization dependent at an oblique incidence. (Bottom) The preferred design: a spherical dome concentric with the center of projection eliminates this effect by creating normal incidence angles.

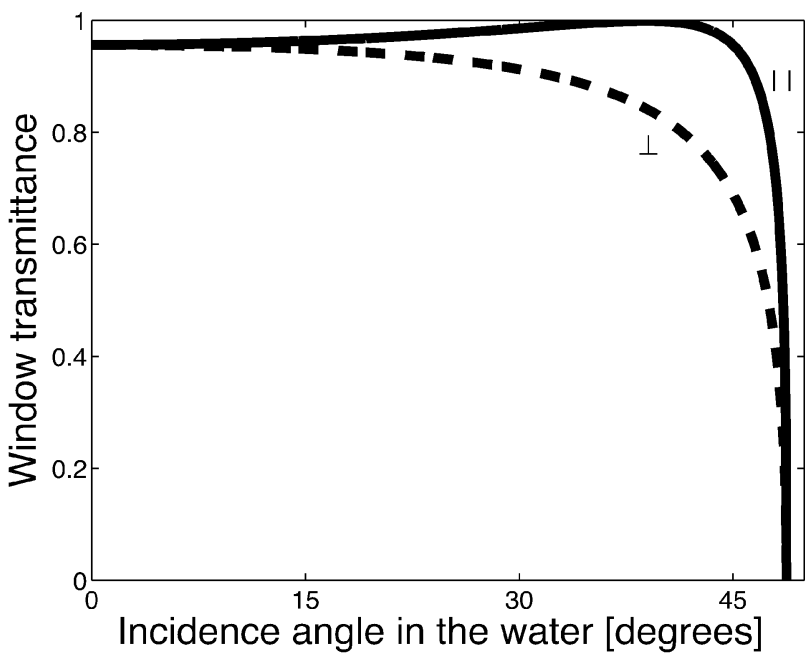

Fig. 22. Transmittance of the polarization components derived from Fresnel equations. These components are in a local coordinate system: they are parallel and perpendicular to the plane created by the chief ray and the window's normal. These are not necessarily the parallel and perpendicular components of the veiling light.

tector is normal to the dome interface. At normal incidence, the transmittance is independent of the linear polarization state [79]. For this reason, we decided to use a dome port [80], [81].

A linear or an inverted circular polarizer? Even if we use a dome port, not all light rays have a normal incidence. One

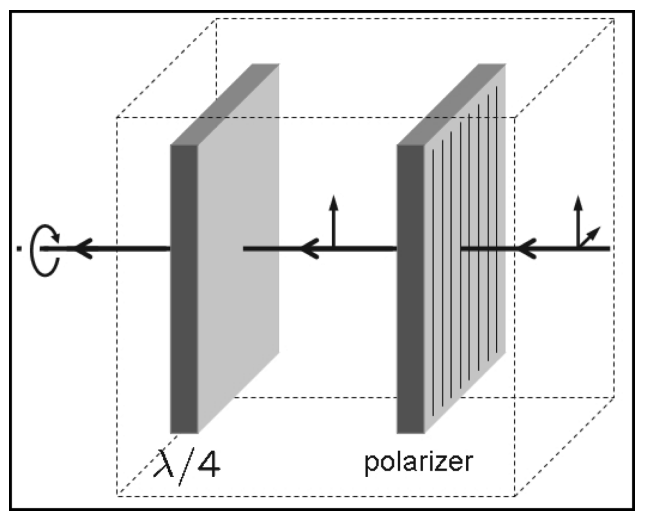

Fig. 23. Inverted circular polarizer composed of a linear polarizer and a $\lambda / 4$ plate. It filters the linear polarization of its input (scene) while it outputs circular polarization.

reason is that the dome may not be precisely concentric with the center of projection. In nonnormal incidence, different polarization components are transmitted differently, affecting the intensity readouts. To reduce this effect, we decided to use an inverted circular polarizer. An inverted circular polarizer (Fig. 23) is composed of a linear polarizer followed by a $\lambda / 4$ plate. It filters the linear polarization of its input (scene) while it outputs circular polarization [79] to the dome. In this case, dome transmittance is invariant to the polarizer angle. We note that circular polarizers are tuned to normal incidence and to a narrow spectral band. Light outside that band or off axis creates elliptical 
polarization. ${ }^{13}$ The port transmittance of elliptical polarization is still less variant to the polarizer angle than when light is partially linearly polarized. So, while this measure helps in minimizing unwanted polarization effects, the other considerations listed above should be employed as well.

\section{ACKNOWLEDGMENT}

The authors are grateful to N. Shashar for helpful discussions, Y. Kahanov for supplying the underwater archaeological pictures (including Fig. 1), Y. Fhiler and N. Geva for help in the dives, and N. Blau for helping with the dives and allowing the use of his photograph of the action (Fig. 8).

\section{REFERENCES}

[1] D. F. Coleman, J. B. Newman, and R. D. Ballard, "Design and implementation of advanced underwater imaging systems for deep sea marine archaeological surveys," in Proc. Marine Technology Society (MTS)/IEEE OCEANS, vol. 1, Providence, RI, 2000, pp. 661-665.

[2] S. Harsdorf, R. Reuter, and S. Tönebön, "Contrast-enhanced optical imaging of submersible targets," in Proc. Int. Society Optical Engineering (SPIE), vol. 3821, San Diego, CA, 1999, pp. 378-383.

[3] J. S. Jaffe, "Computer modelling and the design of optimal underwater imaging systems," IEEE J. Ocean. Eng., vol. 15, no. 2, pp. 101-111, 1990.

[4] D. M. Kocak and F. M. Caimi, "Computer vision in ocean engineering," in The Ocean Engineering Handbook, F. El-Hawary, Ed. Boca Raton, FL: CRC Press, 2001, ch. 4.3.

[5] J. S. Taylor Jr. and L. B. Wolff, "Partial polarization signature results from the field testing of the shallow water real-time imaging polarimeter (shrimp)," in Proc. Marine Technology Society (MTS)/IEEE OCEANS, vol. 1, Honolulu, HI, 2001, pp. 107-116.

[6] L. B. Wolff, "Polarization vision: A new sensory approach to image understanding," Image Vis. Comput., vol. 15, no. 2, pp. 81-93, 1997.

[7] G. L. Foresti, "Visual inspection of sea bottom structures by an autonomous underwater vehicle," IEEE Trans. Syst. Man Cybern., vol. 31, pp. 691-705, Oct. 2001.

[8] L. Botelho-Ribeiro, "Underwater vision method for low-visibility turbulent conditions," in Proc. Marine Technology Society (MTS)/IEEE OCEANS, vol. 2, Honolulu, HI, 2001, pp. 1080-1084.

[9] T. Boult, "Dove: Dolphin omni-directional video equipment," in Proc. Int. Association Science and Technology Development (IASTED) Int. Conf. Robotics and Automation, San Francisco, CA, 2000, pp. 214-220.

[10] F. M. Caimi, B. C. Bailey, and J. H. Blatt, "Spatial coherence methods in undersea image formation and detection," in Proc. Marine Technology Society (MTS)/IEEE OCEANS, Fort Lauderdale, FL, 1996, pp. $40-46$.

[11] J. L. Forand, G. R. Fournier, D. Bonnier, and P. Pace, "LUCIE: A laser underwater camera image enhancer," in Proc. IEEE OCEANS, vol. 3, Victoria, Canada, 1993, pp. 187-190.

[12] G. D. Gilbert and J. C. Pernicka, "Improvement of underwater visibility by reduction of backscatter with a circular polarization technique," Appl. Opt., vol. 6, no. 4, pp. 741-746, 1967.

[13] G. W. Kattawar and M. J. Rakovic, "Virtues of mueller matrix imaging for underwater target detection," Appl. Opt., vol. 38, no. 30, pp. 6431-6438, 1999.

[14] L. J. Mullen and V. M. Contarino, "Hybrid lidar-radar: Seeing through the scatter," IEEE Microw. Mag., vol. 1, no. 3, pp. 42-48, 2000.

[15] V. Murino and A. Trucco, "Underwater computer vision and pattern recognition," Comput. Vis. Image Underst., vol. 79, no. 1, pp. 1-3, 2000.

[16] B. A. Swartz, "Laser range gate underwater imaging advances," in Proc. Marine Technology Society (MTS)/IEEE OCEANS, Brest, France, 1994, pp. 722-727.

[17] A. Ortiz, M. Simo, and G. Oliver, "A vision system for an underwater cable tracker," Mach. Vis. Appl., vol. 13, no. 3, pp. 129-140, 2002.

\footnotetext{
${ }^{13}$ There are some new wide-band and wide-angle retarders [82]. These retarders can be used to form wide-band and wide-angle inverted circular polarizers.
}

[18] J. M. Lavest, F. Guichard, and C. Rousseau, "Multiview reconstruction combining underwater and air sensors," in Proc. IEEE Int. Conf. Image Processing, vol. 3, Rochester, NY, 2002, pp. 813-816.

[19] X. Xu and S. Negahdaripour, "Automatic optical station keeping and navigation of an ROV: Sea trial experiment," in Proc. Marine Technology Society (MTS)/IEEE OCEANS, vol. 1, Seattle, WA, 1999, pp. 71-76.

[20] J. Åhlén and D. Sundgren, "Bottom reflectance influence on a color correction algorithm for underwater images," in Proc. Scandinavian Conf. Image Analysis, Gothenburg, Sweden, 2003, pp. 922-926.

[21] G. Horváth and D. Varjú, "Underwater refraction-polarization patterns of skylight perceived by aquatic animals through Snell's window of the flat water surface," Vis. Res., vol. 35, no. 12, pp. 1651-1666, 1995.

[22] N. Shashar and T. W. Cronin, "Polarization contrast vision in octopus," J. Exp. Biol., vol. 199, no. 4, pp. 999-1004, 1996.

[23] R. D. Ballard, "Ancient Ashkelon," Natl. Geogr., vol. 199, no. 1, pp. 91-93, 2001.

[24] Y. Kahanov and J. Royal, "Analysis of hull remains of the Dor D vessel, Tantura lagoon, Israel," Int. J. Naut. Archeol., vol. 30, no. 2, pp. 257-265, 2001.

[25] K. D. Moore and J. S. Jaffe, "Time-evolution of high-resolution topographic measurements of the sea floor using a 3-D laser line scan mapping system," IEEE J. Ocean. Eng., vol. 27, no. 3, pp. 525-542, 2002.

[26] B. Skerry and H. Hall, Successful Underwater Photography. New York: Amphoto Books, 2002, pp. 25-41.

[27] M. Webster, The Art and Technique of Underwater Photography. Surrey, England, U.K.: Fountain Press, 1998, pp. 19-22, 33-39, 146-149.

[28] D. K. Lynch and W. Livingston, Color and Light in Nature, 2nd ed. Cambridge, U.K.: Cambridge Univ. Press, 2001, ch. 2.4, 2.5, 3.7, 3.16.

[29] F. Cozman and E. Krotkov, "Depth from scattering," in Proc. IEEE Computer Society Conf. Computer Vision and Pattern Recognition, San Juan, PR, 1997, pp. 801-806.

[30] O. Emile, F. Bretenaker, and A. L. Floch, "Rotating polarization imaging in turbid media," Opt. Lett., vol. 21, no. 20, pp. 1706-1708, 1996.

[31] T. KokKeong and J. P. Oakley, "Physics-based approach to color image enhancement in poor visibility conditions," J. Opt. Soc. Amer. A, vol. 18, no. 10 , pp. 2460-2466, 2001.

[32] S. G. Narasimhan and S. K. Nayar, "Vision and the atmosphere," Int. J. Comput. Vis., vol. 48, no. 3, pp. 233-254, 2002.

[33] J. P. Oakley and B. L. Satherley, "Improving image quality in poor visibility conditions using a physical model for contrast degradation," IEEE Trans. Image Process., vol. 7, pp. 167-179, Feb. 1998.

[34] Y. Y. Schechner and N. Karpel, "Clear underwater vision," in Proc. IEEE Computer Society Conf. Computer Vision and Pattern Recognition, vol. I, Washington, DC, 2004, pp. 536-543.

[35] S. M. Christie and F. Kvasnik, "Contrast enhancement of underwater images with coherent optical image processors," Appl. Opt., vol. 35, no. 5, pp. 817-825, 1996.

[36] D. Miyazaki, M. Saito, Y. Sato, and K. Ikeuchi, "Determining surface orientations of transparent objects based on polarization degrees in visible and infrared wavelengths," J. Opt. Soc. Amer. A, vol. 19, no. 4, pp. 687-694, 2002.

[37] Y. Y. Schechner, S. G. Narasimhan, and S. K. Nayar, "Polarization-based vision through haze," Appl. Opt., vol. 42, no. 3, pp. 511-525, 2003.

[38] Y. Y. Schechner, J. Shamir, and N. Kiryati, "Polarization and statistical analysis of scenes containing a semi-reflector," J. Opt. Soc. Amer. A, vol. 17, no. 2, pp. 276-284, 2000.

[39] L. J. Denes, M. Gottlieb, B. Kaminsky, and P. Metes, "AOTF polarization difference imaging," in Proc. Int. Society Optical Engineering (SPIE) Advances in Computer-Assisted Recognition, vol. 3584, Washington, DC, 1999, pp. 106-115.

[40] T. W. Cronin, N. Shashar, R. L. Caldwell, J. Marshall, A. G. Cheroske, and T. H. Chiou, "Polarization vision and its role in biological signaling," Integr. Comp. Biol., vol. 43, no. 4, pp. 549-558, 2003.

[41] G. Horváth and D. Varjú, Polarized Light in Animal Vision. Berlin, Germany: Springer-Verlag, 2004, ch. 14, 25, 26, 28.

[42] S. Johnsen, "Transparent animals," Sci. Amer, vol. 282, no. 2, pp. 80-89, Feb. 2000

[43] J. N. Lythgoe, "The adaptation of visual pigments to the photic environment," in Handbook of Sensory Physiology. Berlin, Germany: Springer-Verlag, 1972, vol. VII/1, ch. 14, pp. 566-603.

[44] M. Michinomae, H. Masuda, M. Seidou, and Y. Kito, "Structural basis for wavelength discrimination in the banked retina of the firefly squid Watasenia scintillans," J. Exp. Biol., vol. 193, no. 11-12, pp. 1-12, 1994.

[45] D. C. Parkyn, J. D. Austin, and C. W. Hawryshyn, "Acquisition of polarized-light orientation in salmonids under laboratory conditions," Anim. Behav, vol. 65, pp. 893-904, 2003. 
[46] M. P. Rowe, E. N. Pugh Jr., J. S. Tyo, and N. Engheta, "Polarizationdifference imaging: A biologically inspired technique for observation through scattering media," Opt. Lett., vol. 20, no. 6, pp. 608-610, 1995.

[47] T. H. Waterman, "Polarization sensitivity," in Handbook of Sensory Physiology. Berlin, Germany: Springer-Verlag, 1981, vol. VII/6B, ch. 3, pp. 281-469.

[48] R. Wehner, "Polarization vision-A uniform sensory capacity?," J. Exp. Biol., vol. 204, no. 14, pp. 2589-2596, 2001.

[49] D.-M. He and G. G. L. Seet, "Divergent-beam lidar imaging in turbid water," Opt. Lasers Eng., vol. 41, no. 1, pp. 217-231, 2004.

[50] J. S. Jaffe, "Sensors for underwater robotic vision: Status and prospects," in Proc. IEEE Int. Conf. Robotics and Automation, Sacramento, CA, 1991, pp. 2759-2766.

[51] M. Levoy, B. Chen, V. Vaish, M. Horowitz, I. McDowall, and M. Bolas, "Synthetic aperture confocal imaging," ACM Trans. Graph., vol. 23, no. 3 , pp. 825-834, 2004

[52] D. N. Sitter Jr. and A. Gelbart, "Laser-induced fluorescence imaging of the ocean bottom," Opt. Eng., vol. 40, no. 8, pp. 1545-1553, 2001.

[53] J. G. Walker, P. C. Y. Chang, and K. I. Hopcraft, "Visibility depth improvement in active polarization imaging in scattering media," Appl. Opt., vol. 39, no. 27, pp. 4933-4941, 2000.

[54] J. N. Lythgoe and C. C. Hemmings, "Polarized light and underwater vision," Nature, vol. 213, no. 79, pp. 893-894, 1967.

[55] S. M. Luria and J. A. S. Kinney, "Linear polarizing filters and underwater vision," Undersea Biomed. Res., vol. 1, no. 4, pp. 371-378, 1974.

[56] G. P. Können, Polarized Light in Nature. New York: Cambridge Univ. Press, 1985, pp. 1-10, 96-99, 131-137, 144-145.

[57] C. D. Mobley, Light and Water: Radiative Transfer in Natural Waters. San Diego, CA: Academic, 1994, ch. 3, 5.

[58] P. C. Y. Chang, J. C. Flitton, K. I. Hopcraft, E. Jakeman, D. L. Jordan, and J. G. Walker, "Improving visibility depth in passive underwater imaging by use of polarization," Appl. Opt., vol. 42, no. 15, pp. 2794-2802, 2003.

[59] E. Gaten, P. M. J. Shelton, and M. S. Nowel, "Contrast enhancement through structural variations in the rhabdoms of oplophorid shrimps," Mar. Biol., vol. 145, no. 3, pp. 499-504, 2004.

[60] L. Peichl, G. Behrmann, and R. H. H. Kröger, "For whales and seals the ocean is not blue: A visual pigment loss in marine mammals," Eur. $J$. Neurosci., vol. 13, no. 8, pp. 1520-1529, 2001

[61] G. G. Rosenthal and M. J. Ryan, "Visual and acoustic communication in non-human animals: A comparison," J. Biosci., vol. 25, no. 3, pp. 285-290, 2000.

[62] N. Shashar, R. Hagan, J. G. Boal, and R. T. Hanlon, "Cuttlefish use polarization sensitivity in predation on silvery fish," Vis. Res., vol. 40, no. 1, pp. 71-75, 2000.

[63] J. S. Tyo, M. P. Rowe, E. N. Pugh Jr., and N. Engheta, "Target detection in optically scattering media by polarization-difference imaging," Appl. Opt., vol. 35, no. 11, pp. 1855-1870, 1996.

[64] W. S. Jagger and W. R. A. Muntz, "Aquatic vision and the modulation transfer properties of unlighted and diffusely lighted natural waters," Vis. Res., vol. 33, no. 13, pp. 1755-1763, 1993.

[65] N. G. Jerlov, Marine Optics, ser. Elsevier Oceanography. Amsterdam, The Netherlands: Elsevier, 1976, pp. 87, 96-100, 151-155.

[66] B. L. McGlamery, "A computer model for underwater camera system," Proc. SPIE, vol. 208, pp. 221-231, 1979.

[67] S. Q. Duntley, "Light in the sea," J. Opt. Soc. Amer, vol. 53, no. 2, pp. 233-241, 1963

[68] K. J. Voss, "Simple empirical model of the oceanic point spread function," Appl. Opt., vol. 30, no. 18, pp. 2647-2651, 1991.

[69] R. C. Henry, S. Mahadev, S. Urquijo, and D. Chitwood, "Color perception through atmospheric haze," J. Opt. Soc. Amer. A, vol. 17, no. 5, pp. $831-835,2000$

[70] S. M. Luria and J. A. S. Kinney, "Underwater vision," Science, vol. 167, no. 924 , pp. $1454-1461,1999$.

[71] E. H. Adelson, "Lightness perception and lightness illusions," in The New Cognitive Neuroscience, 2nd ed. Cambridge, MA: MIT press, 2000 , ch. 24 , pp. 339-351.
[72] M. G. J. Minnaert, Light and Color in the Outdoors (1974). New York: Springer-Verlag, 1983

[73] S. P. Morgan, M. P. Khong, and M. G. Somekh, "Effects of polarization state and scatterer concentration on optical imaging through scattering media," Appl. Opt., vol. 36, no. 7, pp. 1560-1565, 1997.

[74] Y. Y. Schechner and N. Karpel, "Attenuating natural flicker patterns," in Proc. Marine Technology Society (MTS)/IEEE OCEANS, Kobe, Japan, 2004, pp. $1262-1268$.

[75] A. K. Jain, Fundamentals of Digital Image Processing. Englewood Cliffs, NJ: Prentice-Hall, 1989.

[76] Y. Y. Schechner. [Online] http://www.ee.technion.ac.il/ yoav

[77] J. A. Strickrott and S. Negahdaripour, "On the development of an active vision system for 3-d scene reconstruction and analysis from underwater images," in Proc. Marine Technology Society (MTS)/IEEE OCEANS, vol. 1, Halifax, NS, Canada, 1997, pp. 626-633.

[78] S. Zhang and S. Negahdaripour, "3-d shape recovery of planar and curved surfaces from shading cues in underwater images," IEEE J. Ocean. Eng., vol. 27, no. 1, pp. 100-116, 2002.

[79] W. A. Shurcliff and S. S. Ballard, Polarized Light. Princeton, NJ: Van Nostrand, 1964, pp. 98-103, 120-121.

[80] J. M. Lavest, G. Rivers, and J. T. Lapreste, "Dry camera calibration for underwater applications," Mach. Vis. Appl., vol. 13, no. 5-6, pp. 245-253, 2003.

[81] R. Li, H. Li, W. Zou, R. G. Smith, and T. A. Curran, "Quantitative photogrammetric analysis of digital underwater video imagery," IEEE J. Ocean. Eng., vol. 22, no. 2, pp. 364-375, 1997.

[82] S. Guimond and D. Elmore, "Polarizing views," SPIE OE Mag., vol. 4 no. 5 , pp. 26-29, May 2004

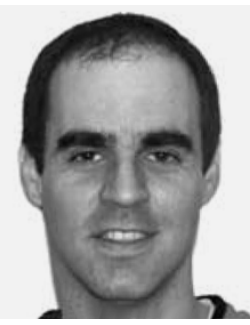

Yoav Y. Schechner received the B.A. and M.Sc. degrees in physics and the Ph.D. degree in electrical engineering from the Technion-Israel Institute of Technology, Haifa, in 1990, 1996, and 1999, respectively.

$\mathrm{He}$ is currently the Head of the Hybrid Imaging Laboratory, Department of Electrical Engineering, Technion. His research is focused on the use of optics and physics in imaging and computer vision. In particular, he is interested in multimodal sensing.

Mr. Schechner was the recipient of the Wolf Foundation Award for Graduate Students in 1994, the Gutwirth Special Distinction Fellowship in 1995, the Israeli Ministry of Science (Eshkol) Distinction Fellowship and the Ollendorff Award in 1998, the Schwarz Foundation Award in 1999, and the Morin Fellowship in 2000-2002. He is now a Landau Fellow - supported by the Taub Foundation, and an Alon Fellow.

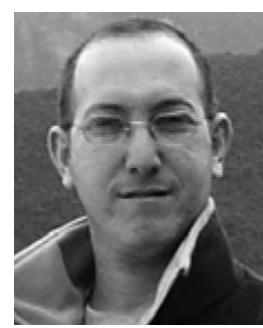

Nir Karpel received the B.Sc. degree in mechanical engineering and the M.Sc. degree in electrical engineering from the Technion-Israel Institute of Technology, Haifa, in 2000 and 2005, respectively.

His research involves optics and computer vision. 